\title{
IoT Traffic Management and Integration in the QoS Supported Network
}

\author{
Basim K. J. Al-Shammari ${ }^{\circledR}$, Graduate Student Member, IEEE, Nadia Al-Aboody ${ }^{\circledR}$, Member, IEEE, \\ and Hamed S. Al-Raweshidy, Senior Member, IEEE
}

\begin{abstract}
This paper proposes: 1) a traffic flow management policy, which allocates and organizes machine type communication (MTC) traffic flows network resources sharing within evolved packet system (EPS); 2) an access element as a wireless sensor network gateway for providing an overlaying access channel between the machine type devices and EPS; and 3) it addresses the effect and interaction in the heterogeneity of applications, services and terminal devices, and the related quality of service (QoS) issues among them. This paper overcomes the problems of network resource starvation by preventing deterioration of network performance. The scheme is validated through simulation, which indicates the proposed traffic flow management policy outperforms the current traffic management policy. Specifically, simulation results show that the proposed model achieves an enhancement in QoS performance for the MTC traffic flows, including a decrease of $99.45 \%$ in packet loss rate (PLR), a decrease of $99.89 \%$ in packet end to end (E2E) delay, a decrease of $99.21 \%$ in packet delay variation (PDV). Furthermore, it retains the perceived quality of experience of the real time application users within high satisfaction levels, such as the voice over long term evolution service possessing a mean opinion score (MOS) of 4.349 and enhancing the QoS of a video conference service within the standardized values of a 3GPP body, with a decrease of $85.28 \%$ in PLR, a decrease of $85 \%$ in packet E2E delay and a decrease of $\mathbf{8 8 . 5 \%}$ in PDV.
\end{abstract}

Index Terms-Application quality of service (AQoS), end to end (E2E) delay, gateway, human type communication (HTC), Internet of Things (IoT), jitter, machine type communication (MTC), network QoS (NQoS), quality of experience (QoE), QoS class identifier (QCI), traffic policy.

\section{NOMENCLATURE}

\section{List of Abbreviations}

$\begin{array}{ll}3 \mathrm{G}, 4 \mathrm{G}, 5 \mathrm{G} & \text { 3rd, 4th, 5th generation. } \\ \text { AC } & \text { Admission control. } \\ \text { ARP } & \text { Allocation and retention priority. } \\ \text { AQoS } & \text { Application QoS. } \\ \text { BS } & \text { Base station. } \\ \text { CoAP } & \text { Constrained application protocol. } \\ \text { DL } & \text { Downlink. } \\ \text { DSCP } & \text { Differentiated services code point. } \\ \text { E2E } & \text { End to end. }\end{array}$

Manuscript received May 25, 2017; revised November 19, 2017; accepted December 14, 2017. Date of publication December 19, 2017; date of current version February 9, 2018. (Corresponding author: Basim K. J. Al-Shammari.)

The authors are with the Department of Electronic and Computer Engineering, Brunel University London, London UB8 3PH, U.K. (e-mail: basim.al-shammari@brunel.ac.uk).

Digital Object Identifier 10.1109/JIOT.2017.2785219

\begin{tabular}{|c|c|}
\hline eNB & evolved NodeB. \\
\hline EPS & Evolved packet system. \\
\hline $\mathrm{UE}$ & User equipment. \\
\hline FTP & File transfer protocol. \\
\hline GBR & Guaranteed bit rate. \\
\hline $\mathrm{H} 2 \mathrm{H}$ & Human-to-human. \\
\hline HTC & Human type communication. \\
\hline HTD & Human type device. \\
\hline IoT & Internet of Things. \\
\hline IP & Internet protocol. \\
\hline KPI & Key performance indicator. \\
\hline KQIs & Key quality indicators. \\
\hline LPWAN & Low power WAN. \\
\hline LTE & Long term evolution. \\
\hline LTE-A & LTE Advanced. \\
\hline LTE-APro & LTE-A proximity. \\
\hline $\mathrm{M} 2 \mathrm{M}$ & Machine to machine. \\
\hline MAC & Medium access control. \\
\hline MBR & Maximum bit rate. \\
\hline $\mathrm{MC}$ & Mission critical. \\
\hline $\mathrm{MCN}$ & Mobile cellular networks. \\
\hline MOS & Mean opinion score. \\
\hline MTC & Machine type communication. \\
\hline MTD & Machine type devices. \\
\hline NAS & Non access stratum. \\
\hline NB-IoT & Narrow band IoT. \\
\hline NGBR & Non-guaranteed bit rate. \\
\hline NGN & Next generation network. \\
\hline NQoS & Network QoS. \\
\hline PDB & Packet delay budget. \\
\hline $\mathrm{PDCCH}$ & Physical downlink control channel. \\
\hline PDV & Packet delay variation. \\
\hline $\mathrm{PDN}$ & Packet data network. \\
\hline PCEF & Policy control enforcement function. \\
\hline PCC & Policy charging and control. \\
\hline PLR & Packet loss rate. \\
\hline PRACH & Physical random access channel. \\
\hline PS & Packet switching. \\
\hline PUSCH & Physical uplink shared channel. \\
\hline QoE & Quality of experience. \\
\hline QoS & Quality of service. \\
\hline QCI & QoS class identifier. \\
\hline RAN & Radio access network. \\
\hline SDF & Service data flow. \\
\hline $\mathrm{SN}$ & Sensor node. \\
\hline TFT & Traffic flow template. \\
\hline
\end{tabular}


TTI Transmission time interval.

UL Uplink.

VoLTE Voice over LTE.

WAN Wide area network.

WSN Wireless sensor network.

\section{INTRODUCTION AND RELATED WORK}

$\mathbf{M}$ TC (or M2M communication) introduces the concept of enabling networked devices (wireless and/or wired), electronic devices, dubbed MTC devices and/or communication from MTC devices to a central MTC server or a set of MTC servers, for exchanging data or control without human interference. This includes the mechanisms, algorithms, and the technologies used to enable MTC communication. Which involves connecting large number of devices or piece of software with the creation of the IoT. MTC developments are driven by the unlimited potential of IoT everyday applications, as a result from the connected objects to grow more intelligent. These applications presented in the form of smart grids, healthcare systems, smart cities, building automation, and many other. Such systems can be implemented through the use of WSN, within which the utilization of cooperating devices can achieve intelligent monitoring and management.

IoT presents a massive ecosystem of new elements, applications, functions, and services. Existing transmits of MTC traffic flows for IoT devices run over wireless WAN, $3 \mathrm{G}$ and $4 \mathrm{G}$ networks. However, $5 \mathrm{G}$ is expected to become the main platform for meeting the requirements of IoT traffic demand in the very near future. EPS protocols and architectures were aimed and enhanced to handle HTC traffic flows. With the anticipated mass deployment of MTDs, there are concerns from the network operators side, the service provider side and from the application consumers perspective. The fear is that the network ecosystem may become exhausted and/or the available network resources will not be able to meet AQoS traffic flow requirements for both HTC and MTC traffic flows simultaneously. Moreover, MTC traffic characteristics of IoT devices are different from those of HTC and can augment the risk of network performance deterioration.

Future networks will gradually be converted into integrated entities with high broadband and large-scale traffic due to the interconnection of social medias, virtual systems, and physical networks [1]. It is estimated that between 50 to 100 billion MTC devices will be connected to the Internet by 2020 [2], [3] and that the majority of these devices will be run through cellular wireless networks and WSNs. However, cellular networks were mainly designed for $\mathrm{H} 2 \mathrm{H}$ communication, where the UL resource allocation is less than the DL. In contrast, the M2M traffic could produce more data in the UL channels, which can lead to a deterioration of the human QoE or could overload the network. To overcome this challenge, an efficient resource allocation model can be used to take full advantage of the opportunities created by a global MTC market over cellular networks. The 3GPP and the IEEE standardization bodies have initiated their working groups for facilitating such applications through various releases of their standards [4].
LTE-A is very close to reach the technologically possible efficiency limits in the currently available bands, according to 3GPP, hence, it is expected that LTE will remain as the baseline technology for wide area broadband coverage also in the 5G era. However, integrating M2M communication with LTE-A can have a dramatic negative impact on LTE network performance in terms of QoS and throughput [5]. Consequently, 3GPP will continue working on enhancing LTE to make it more suitable for $\mathrm{M} 2 \mathrm{M}$ communication from a radio perspective as well as from a service delivery one and hence, mobile M2M traffic must not be neglected.

Costantino et al. [6] evaluated the performance of an LTE gateway using the CoAP, with the M2M traffic patterns and the network configurations being presented through simulations. The authors argued that the traffic patterns highly depending on the application used in the terminal devices, however, they did not describe or justify their choices. Their work focused on the effect of signals interference, in radio access node, related to the number of connected MTDs.

Lo et al. [7] studied the impact of data aggregation for M2M on QoS in an LTE-A network. In their work, they utilized a relay node as an aggregation and entry point of MTD traffic to improve LTE-A UL efficiency. Specifically, their work was based on tunneling and aggregation of the MTDs, which were related to a specific destination server or node, via the relay node tunnel entry point and by considering the priority of the class of service. Whilst a significant reduction in signaling overhead was achieved, there was an increase in the delay of the received packet at the destination point and an increase in the number of aggregated MTDs at the entry point.

There have been several attempts at delivering M2M communication integration with LTE-A. The current state of standardization efforts in M2M communication and the potential M2M problems, including physical layer transmissions were discussed in [8], who proposed a solution to provide QoS guarantees by facilitating M2M applications with hard timing constraints. Moreover, the features of M2M communication in 3GPP, an overview of the network architecture, random access procedure (RAP), and radio resources allocations were provided. Kang et al. [9] considered one service and proposed a cooperative strategy for energy efficient MTD video transmission with a good NQoS. They achieved promising results, however, in the future, networks with different service and network components will be used in a heterogeneous environment. A relay node was proposed by Marwat et al. [10] to facilitate the integration of M2M, with the proposed work being focused on a relaying feature in LTE-A. Zhang et al. [11] examined the network convergence between MCNs and WSNs, proposing that the mobile terminals in MCN should act as both SNs and gateways for WSN in the converged networks. Their work neither guaranteed the E2E connectivity nor specified RANs technology involved. There has been no work, as yet, to the best of our knowledge, proposing an entirely heterogeneous model that serves different networks with different services.

Another strand of research considered the radio access interface, including the new LPWANs, such as NB-IoT in the licensed spectrum as well as its competitors LoRa, SigFox, 
and Ingenu in the unlicensed spectrum [12]. The unlicensed networks have been deployed around the world since the first quarter of 2016, which motivated 3GPP to introduce NB-IoT in the second quarter of 2016. NB-IoT uses the guard bands in the $3 \mathrm{G}$ and $4 \mathrm{G}$ licensed frequency spectrum bands to provide a dedicated radio access for IoT devices within the radio spectrum of the currently deployed $3 \mathrm{G}$ and $4 \mathrm{G}$ RANs [13]. In the same context, the authors in [14]-[16] presented a connection-less integration of low rate IoT traffic within the PRACH frequency-time slot of LTE access technology. The previously listed technologies and research attempts did not address the effect of the heterogeneity of MTC application traffic and related QoS performance of HTC application when the network ecosystem resources have to be shared in such a way. This paper's main contributions are as follows.

1) Proposing an access WSN gateway to provide access and connectivity for MTD traffic within an LTE-APro eNB license spectrum radio interface.

2) Proposing a traffic flow management policy to define the existence of IoT traffic with $\mathrm{H} 2 \mathrm{H}$ traffic over an EPS network and provide an acceptable level of NQoS for MTC traffic flows, whilst preserving the QoE of HTCs.

3) Introducing specific QCIs, which are elected to classify the MTC traffic flows, whilst taking into account the heterogeneity of SNs services and applications, over an EPS network, in order to discriminate the treatment of EPS from the NQoS point of view from the standard QCIs of HTC traffic flows.

The rest of this paper is organized as follows: the next section presents a background to MTC traffic characteristics and QoS in EPS provision and management. Section III presents the design of the proposed traffic flow management model. Section IV introduces the simulation setup and presents the main results. A performance discussion is presented in Section V. Finally, Section VI concludes this paper and also puts forward suggestions for future work.

\section{BACKGROUND}

\section{A. MTC Traffic Characteristics}

MTC terminals or SN can communicate with one or many servers and their traffic characteristics have been discussed in detail in [17]. They can be considered as data centric communication for sending constant size packets toward server [18], [19]. Their traffic main characteristics are as follows.

1) SDF of MTC traffic is typically from the SN toward PDN, which will be more dominant in the UL direction. Whereas HTC's SDF traffic depends on the service type and its AQoS demands during the call session or service request.

2) In much of MTC applications traffic, the SN harvests a small amount of information. This can be transferred at a specific data rate, which matches the applications needs.

3) In the NGN, the MTC traffic has a share of the network resources and they will be not limited just for HTC. The MTC are going to join the use of licensed spectrum and take a share of the mobile broadband network access resources.

4) In general, MTC traffic is delay tolerant and for the most part, it can be considered as having low priority. 3 GPP in release 10 introduces delay tolerant access in the RAP as part of control plane signaling to provide a lower priority in the PRACH for MTC traffic than HTC Traffic [16]. However, some MTC traffic is excluded from using this procedure, such as industrial control systems, fire alarms, and live monitoring, as they are need to be transmitted with high priority. LTE-A release 13 introduces new QCI to provide a high priority SDF for emergency service application use cases [20], [21].

5) SN data transmission is irregular and depends on many factors, which relate to the WSN type and deployment pattern. This irregularity in traffic generation can produce high traffic peaks, which could lead to starvation in the network resources, specifically in the wireless access part.

The basic concern in MTC within the NGN is how to provide the required NQoS levels or KPIs, which will meet the AQoS of MTC traffic or KQIs, with a massive deployment of SNs, while guaranteeing high levels of expected and perceived quality or QoE of real time application users, simultaneously.

In order to integrate the IoT traffic in the LTE-A standard EPS resources need to be used. Consequently, it is necessary to provide a specific NQoS for MTCs applications traffic so as to avoid the substantial impact on the performance of HTC traffic, such as voice, video, and file transfer and to keep the QoE at a satisfaction level.

\section{B. QoS in EPS Provision and Management}

Applications traffic, including that of HTC and MTC in PS networks has specific AQoS characteristics and demands. They require specific levels of NQoS parameters, which can be described in terms of PLR packet one-way E2E delay, packet jitter at the end point, and link throughput at the IP layer [22], [23]. Packet jitter refers to the variation in the time change between two consecutive packets leaving the source node with time stamps $t 1$ and $t 2$; they are played back at the destination node at $t 3$ and $t 4$, with the jitter being calculated as [24]

$$
\text { Jitter }=(t 4-t 3)-(t 2-t 1) .
$$

In EPS, these diverse NQoS quantitative performance parameters are provisioned via a standardized and effective mechanism, which is a class-based EPS QoS concept [25]. This mechanism allows service providers to deliver service differentiation via NQoS control in the access and backhaul networks, thereby solving the issue of over-provisioning of the valuable network resources.

NQoS control mechanism-based service differentiation is performed on the EPS bearer level, with this bearer being a logical unidirectional connection between PDN-GW and UE as well as it being the core element of NQoS control treatment in EPS. The EPS bearer type and level define the SDFs QoS requirements among all the EPS nodes. EPS introduces two 
TABLE I

STANDARDIZED QCI CHARACTERISTICS

\begin{tabular}{|c|c|c|c|l|l|}
\hline QCI & Resource Type & Priority & PDB msec & PLR & Service \\
\hline 0,255 & \multicolumn{5}{|c|}{ Reserved } \\
\hline 1 & GBR & 2 & 100 & $10^{-2}$ & Conversational Voice \\
\hline 2 & GBR & 4 & 150 & $10^{-3}$ & Conversational Video \\
\hline 3 & GBR & 3 & 50 & $10^{-3}$ & Real Time Gaming \\
\hline 4 & GBR & 5 & 300 & $10^{-6}$ & Non-Conversational Video (Buffered Streaming) \\
\hline 65 & GBR & 0.7 & 75 & $10^{-2}$ & Mission Critical user plane Push To Talk voice (MCPTT) \\
\hline 66 & NGBR & 2 & 100 & $10^{-2}$ & Non-Mission-Critical user plane Push To Talk voice \\
\hline 5 & NGBR & 1 & 100 & $10^{-6}$ & IMS Signalling \\
\hline 6 & NGBR & 6 & 300 & $10^{-6}$ & Video (Buffered Streaming) TCP-Based \\
\hline 7 & NGBR & 7 & 100 & $10^{-3}$ & Voice, Video (Live Streaming), Interactive Gaming \\
\hline 8 & NGBR & 8 & 300 & $10^{-6}$ & Video (Buffered Streaming) TCP-Based \\
\hline 9 & NGBR & 9 & 300 & $10^{-6}$ & Video (Buffered Streaming) TCP-Based, default bearer \\
\hline 69 & NGBR & 0.5 & 60 & $10^{-6}$ & Mission Critical delay sensitive signalling \\
\hline 70 & NGBR & 5.5 & 200 & $10^{-6}$ & Mission Critical Data \\
\hline $10-64,67-68,71-127$ & \multicolumn{3}{|l|}{} \\
\hline $128-254$ & & & \\
\hline
\end{tabular}

specified bearers: 1) default bearer and 2) dedicated bearer. The former is initiated when the UE attaches to the network and remains activated until it detaches. The resource type of the default bearer is NGBR and it supports the best effort QoS level [20]. While the dedicated bearer is created dynamically on demand when the terminal device requests a service or access to services that require a higher QoS level than the default bearer, such as conversational voice or conversational video. The QoS of the EPS bearer is controlled by using a set of QoS's EPS parameters including the following.

1) Resource Types: It defines the bandwidth allocation for the bearer, there being two types, GBR bearer and NGBR. The GBR EPS bearer has a guaranteed minimum bandwidth (bit rate), while the NGBR EPS bearers bandwidth is not assured. NGBR EPS bearer resource allocation depends only on the available resources remaining after the completion of the GBR EPS bearer resource assignment process.

2) QCI: The QoS level of the bearer is assigned to one and only one QCI, which is defined in (3GPP PCC architecture) as a reference between UE and the PCEF, to indicate different IP packet QoS characteristics. It characterizes the SDF in the UL and DL directions by resource type, packet priority handling, PLR and PDB. The QCI comes from the mapping process of the DSCP values that are specified in the IP packet header. The nodes within EPS depend on the QCI value to translate the demand for AQoS. This AQoS to NQoS mapping is to ensure that the packet flow carried on a specific bearer receives the minimum level of QoS in multiuser multiservice networks [20], [25]. In LTE the QCI is an integer value that varies from 0 to 255 , the octet number 3 in EPS QoS information element [20]. While LTE Release 8 specifies nine standard QCI values varying from 1 to 9 [26], LTE-A Release 13 standardized thirteen different QoS levels, with four new QCI values having been added to be used in MC services for emergency services communication, as shown in Table I [20]. QCI value assignment is performed from the network side upon request from an application running on the terminal device.

3) ARP: It is used for call $\mathrm{AC}$, which is an integer value ranging from 1 (highest priority) to 15 (lowest priority) [27]. The ARP value, from the control plane perspective, sets the priority level and treatment for the EPS bearer. It permits the EPS control plane to exercise differentiation regarding control of the bearers setting up and retention. Furthermore, it contains information about bearer pre-emption capability and bearer pre-emption vulnerability. The level of importance of resource request is defined by ARP and used for AC. Table II lists examples of ARP and related QCI values [28]. Whilst data plane traffic packets handling, among the EPS nodes and links, is carried by an associated bearer, as identified by QCI.

4) GBR and MBR: These parameters are used for a GBR dedicated EPS bearer. GBR specifies the minimum provision and assured bit rate, while MBR sets the limit of the MBR allowed within the network. If the dedicated EPS bearer reaches the specified MBR, any new arriving packets will be discarded [27].

5) Access Point Name-Available MBR: This parameter is applied at UE for UL traffic only and it is utilized at PDN-GW for both DL and UL traffic. It controls the total bandwidth of all NGBR EPS bearers [27].

6) UE-Available MBR: It is applied by eNB only, which specifies the maximum allowable bandwidth for all NGBR EPS bearers in the UL and DL directions [27]. 
TABLE II

EXAMPLE OF QCI TO ARP MAPPING

\begin{tabular}{|c|c|c|c|c|c|c|c|c|}
\hline \multicolumn{2}{|c|}{ *ARP } & \multicolumn{2}{|c|}{$* \mathbf{Q C I}$} & \multirow{2}{*}{$\begin{array}{l}\text { Pre-emption } \\
\text { capability }\end{array}$} & \multirow{2}{*}{$\begin{array}{l}\text { Pre-emption } \\
\text { vulnerability }\end{array}$} & \multirow{2}{*}{$\begin{array}{c}\text { Resource } \\
\text { Type }\end{array}$} & \multirow{2}{*}{ Example Services } & \multirow{2}{*}{$\begin{array}{c}\text { Typical Associated } \\
\text { Protocols }\end{array}$} \\
\hline Index & $\begin{array}{c}\text { Bearer } \\
\text { Priority }\end{array}$ & Index & $\begin{array}{c}\text { Packet } \\
\text { Priority }\end{array}$ & & & & & \\
\hline 1 & 1 & 1 & 2 & Yes & No & GBR & Conversational Voice & UDP, SIP, VoIP \\
\hline 2 & 2 & 2 & 4 & Yes & No & GBR & Conversational Video (live streaming) & UDP, RTSP \\
\hline 3 & 3 & 3 & 3 & Yes & No & GBR & Real Time Gaming & UDP, RTP \\
\hline 4 & 4 & 4 & 5 & Yes & No & GBR & Conversational Video (hi-definition) & UDP, RTSP \\
\hline 5 & 5 & 5 & 1 & Yes & Yes & NGBR & IMS signalling & TCP, RTP \\
\hline 6 & 5 & 6 & 6 & Yes & Yes & NGBR & Video (buffered streaming), TCP applications & TCP, FTP \\
\hline 7 & 6 & 7 & 7 & Yes & Yes & NGBR & Voice, Video Live Streaming, Interactive Gaming & TCP, HTTP, VoIP \\
\hline 8 & 6 & 8 & 8 & Yes & Yes & NGBR & Video (buffered streaming), TCP applications & TCP, SMTP, POP \\
\hline 9 & 8 & 9 & 9 & Yes & Yes & NGBR & Video (buffered streaming), TCP applications & TCP, FTP, IMAP \\
\hline 10 & 15 & 5 & 9 & No & Yes & NGBR & UDP based applications & UDP, SNMP \\
\hline 11 & 14 & 6 & 9 & No & Yes & NGBR & UDP based applications & UDP, SNMP, POP \\
\hline 12 & 13 & 7 & 9 & No & Yes & NGBR & UDP based applications & UDP, RTP \\
\hline 13 & 11 & 8 & 9 & No & Yes & NGBR & UDP based applications & UDP \\
\hline 14 & 9 & 9 & 9 & No & Yes & NGBR & UDP based applications & UDP, RTP \\
\hline 15 & 1 & 1 & 2 & Yes & No & GBR & UDP based applications & UDP,SIP, VoIP \\
\hline
\end{tabular}

\section{PROPOSED MODEL}

\section{A. Integration of MTC Traffic Into EPS and Traffic Flow Management Policy}

In order to integrate the IoT traffic in the LTE-A standard, EPS resources need to be used. Consequently, to avoid the substantial impact on the performance of HTC traffic, such as voice, video, HTTP service, and FTP service, a specific NQoS for MTCs applications traffic flows needs to be provided to keep the QoE of real time application users within a satisfaction level. A policy for traffic flow management is proposed in this paper for defining MTC traffic flows over the EPS network, that differentiates the treatment of EPS nodes and links from the NQoS point of view for the different MTC traffic flows and separates the standard HTC traffic flows from MTC ones. The need for a new policy emerged from the drawbacks of the current traffic flow management policy, one which does not differentiate between MTC and HTC traffic flows from a network resources sharing point of view.

Lemma: The MTC traffic flows and HTC traffic flows compete to take shares of the available network resources.

Proof: Let us consider the total available network resources related to the network capacity is $R$, which should be assigned to traffic flows that are transported through the network in each TTI. If the allocated network resources per traffic flow $j$ are $r_{j}$ and the total number of traffic flows is $k$, then the total allocation can be expressed as

$$
\sum_{j=1}^{k}\left(r_{j}\right) \leq R
$$

where, $R=(0, C]$ and $C$ is the maximum capacity of the network from a network resources point of view. The network resource $r_{j}$ can be assigned to the MTC or HTC traffic flow. If an MTC traffic flow of a single MTD $i$ has a share of network resources in each TTI of $\mathrm{IoT}_{i}$, then the number of the network resources assigned to all MTC traffic flows that come from MTDs can be expressed as

$$
\sum_{i=1}^{m}\left(\operatorname{IoT}_{i}\right) \leq S
$$

where, $m$ is the maximum number of the handled MTC traffic flows and $S=(0, R]$. Under the same consideration, the HTC traffic flow of a single HTD $n$ has a share of network resources in each TTI of $H_{n}$, therefore the number of network resources assigned to HTC traffic flows come from HTDs can be expressed as

$$
\Sigma_{n=1}^{l}\left(H_{n}\right) \leq T
$$

where, $l$ is the maximum number of the handled HTC traffic flows and $T=(0, R]$. From (1)-(3) we can deduce that

$$
k=m+l
$$

and

$$
R=S+T
$$

and

$$
\Sigma_{j=1}^{k}\left(r_{j}\right)=\Sigma_{i=1}^{m}\left(\operatorname{IoT}_{i}\right)+\Sigma_{n=1}^{l}\left(H_{n}\right) \leq R
$$

where, the lower and upper boundaries of $\operatorname{IoT}_{i}$ and $H_{n}$ in (7) are $(0, R]$, and the network resources allocation in the evolvedterrestrial RAN (E-UTRAN) network part and evolved packet core (EPC) is mainly based on the bearer's ARP and QCI values as well as the associated NQoS values, which are mapped from the AQoS's DSCP value. As expected in the NGN, the MTC/IoT traffic flows will potentially have a share with HTC traffic flows, therefore the network resources could be assigned to the HTC traffic flows if their bearers are higher (and/or) they 
have network supported ARPs and QCIs comparative to MTC traffic flows bearers, and vice versa. If the MTCs' traffic flow bearers get a resource allocation higher than those of HTC, without determining the management of network resources sharing among them, this will produce some serious issues regarding the offered NQoS and users' QoE. This deterioration in network performance will be clear in NGBR HTC applications, such as FTP and HTTP services.

A proposed solution for the issue of NQoS deterioration is presented here by isolating the available network resources $(R)$, which takes the form of adaptive shared $(\mathcal{R})$ network resources between the MTC network resources share $(\mathcal{S})$ and HTC network resources share $(\mathcal{T})$, by means of two disjoint sets. The size of the two shares $(\mathcal{S}$ and $\mathcal{T})$ is a percentage of available $(\mathcal{R})$, which can be adaptively adjusted based on a dynamic change in the network resources loading share, according to traffic flows conditions. Furthermore, the percentage can be customarily set on a static basis for supporting HTC traffic flows over MTC traffic flows and to reduce the impact of increased network signaling overhead between the E-UTRAN network part and EPC. Therefore, (6) can be rewritten as

$$
\begin{aligned}
\mathcal{R} & =\mathcal{S}+\mathcal{T} \\
\mathcal{S} & =\beta \mathcal{R} \\
\mathcal{T} & =\alpha \mathcal{R} \\
\beta+\alpha & =1
\end{aligned}
$$

where, $\alpha$ is the percentage of network resources share allocated/assigned to the HTC traffic flows and the remaining network resources percentage is $\beta$. For adaptive and dynamic operation, $\alpha$ and $\beta$ are set as a function of AQoS demands to ensure the provision of NQoS in terms of network resources for all traffic flows in the network nodes and transmission links. The EPS system primarily depends on the bearers settings to provide the required NQoS metrics and to cover the needs of AQoS represented by the Terminal Type and DSCP. From 11, $\alpha$ 's and/or $\beta$ 's can be used as tuning parameters to provide the required percentage value of network resources share, which are the bearer's setting, binding and modification parameters. In the $\mathrm{CN}$ and precisely in the policy and charging rule function (PCRF), PCEF, and mobility management entity functions units, these parameters as NQoS are Resource Type, ARP, QCI, PDB, and PLR. Then, it is possible to demonstrate $\alpha$ and $\beta$ as a function of AQoS and/or NQoS. Furthermore, they can be a function of the aforementioned AQoS needs and NQoS provided (from network side) to cover/meet the required performance and to optimize network resources utilization as

$$
\begin{aligned}
\alpha \& \beta & =f(\text { AQoS, NQoS }) \\
& =f(\text { TerminalType, DSCP, ResourceType, ARP, QCI } \\
& \text { PDB, PLR })
\end{aligned}
$$

where, (TerminalType, DSCP) represent the AQoS requirements and (ResourceType, ARP, QCI, PDB, PLR) represent the NQoS offered.

The dynamic variations of $\alpha$ and $\beta$ in every TTI can be deployed in EPC individually to set traffic policy management into a flavor of terminal device type user (human or
TABLE III

Proposed Estimated Values of $\alpha, \beta$ And Associated NETWORK RESOURCES SHARE

\begin{tabular}{|c|c|c|}
\hline Function & $\begin{array}{c}\text { Percentage Share of } \\
\text { over whole Network } \\
\text { Resources }\end{array}$ & $\begin{array}{c}\text { Resource Type } \\
\text { Percentage (sub share) } \\
\text { from each main Share }\end{array}$ \\
\hline$\alpha$ & $80 \%$ & $67 \%$ GBR \\
& $20 \%$ & $33 \%$ NGBR \\
\hline$\beta$ & & $60 \%$ GBR \\
& & $40 \%$ NGBR \\
\hline
\end{tabular}

machine) and the required network resources for the needs of the users' traffic flows, according to (9) and (10). Traffic management policy is realized via an active PCC rule setting or can be applied according to the local policy configuration in the network gateways or from the PCRF network core elements.

The aforementioned PCC rule setting is presented as an adaptive algorithm conferring to the network traffic flows. Another methodology can be interpreted from the previous analysis, for the setting of control mechanism of traffic flow management policy. It consists of two components represented by $\alpha$ and $\beta$. Each one can work with a distinct approach regarding the network resources allocation, separately within its allowable limit, such as optimization and scheduling of network resources usage over different parts of the network. This approach sets a traffic flow management policy by defining a fixed network resource share between HTC and MTC traffic flows. Equation (12) can be rewritten to extract and set the related AQoS and NQoS metrics as follows:

$$
\begin{aligned}
& f^{-1}(\alpha)=(\text { HTCTerminal, DSCP, ARP, QCI, PDB, PLR) } \\
& f^{-1}(\beta)=\text { (MTCTerminal, DSCP, ARP, QCI, PDB, PLR). }
\end{aligned}
$$

Fig. 1 shows the flowchart for setting up the traffic flow management policy in each TTI, or it can be built on a specific duty cycle time basis, whereby the procedure is matched with the TTI of EPS to be mixed with the EPS resource allocation policy. The flowchart starts by checking the available network resources and then checks for traffic flow requests of the HTC and MTC terminals. In Fig. 1(a), the dynamic adaptive network resource share policy has been applied, whereas in Fig. 1(b) a fixed network resource share policy has been applied on a specific HTC terminals network resource share of $\alpha \%$. A specific sort of function is used to match AQoS needs with the NQoS available and to serve the GBR bearers as well as the NGBR bearers for HTC terminal and MTC terminal traffic flows, simultaneously, based on the $\mathcal{T}$ and $\mathcal{S}$ shares.

An estimate and synthetic values set is listed in Table III, for $\alpha$ and $\beta$, according to the traffic density pattern from the productive realistic network scenarios proposed in [18] and [29].

There are three main types of MTC traffic, real time, delay sensitive, and delay tolerant. The proposed QCIs aimed at 


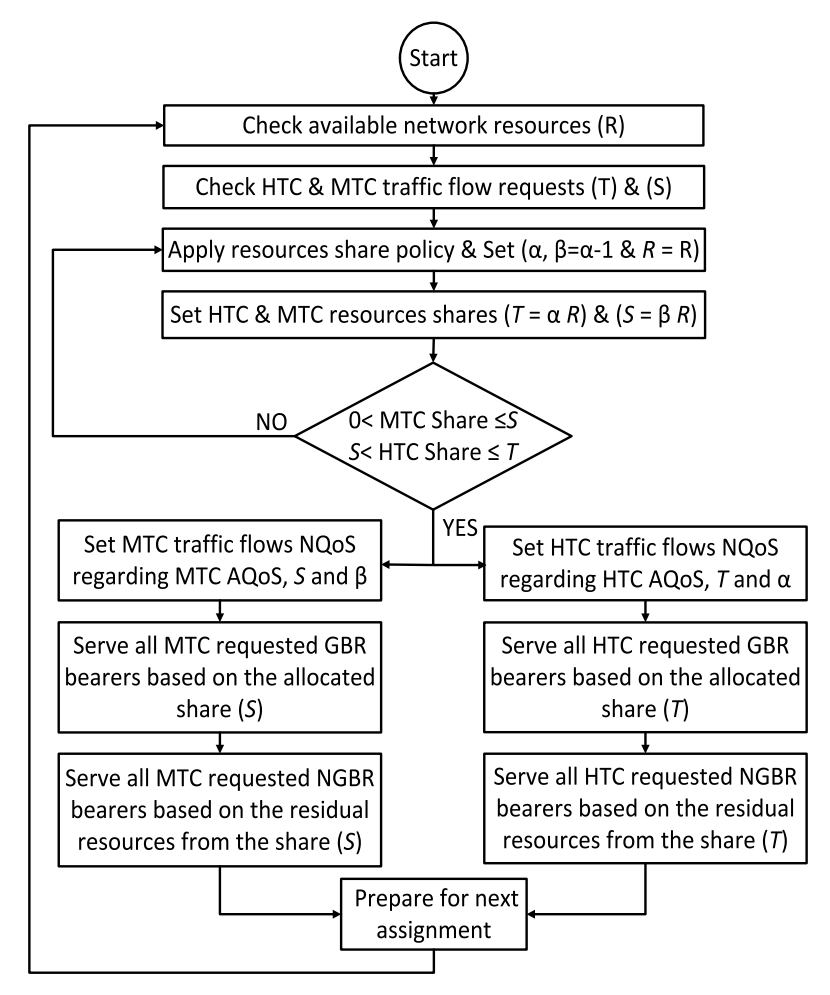

(a)

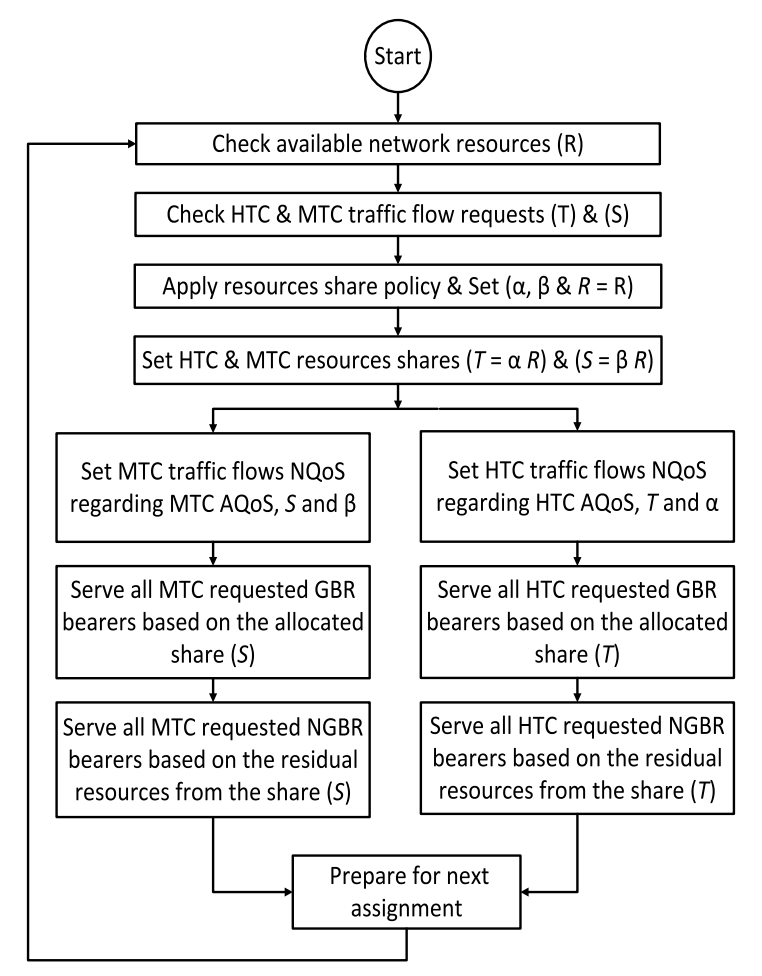

(b)

Fig. 1. Traffic flow management policy setting. (a) Dynamic network resources share policy. (b) Fixed network resources share policy.

TABLE IV PROPOSED MTC QCIS

\begin{tabular}{|c|c|c|c|c|c|}
\hline QCI & Resource Type & Priority* & PDB msec & PLR \% & $\begin{array}{c}\text { Example } \\
\text { services }\end{array}$ \\
\hline 71 & GBR & 1 & 100 & $10^{-3}$ & $\begin{array}{c}\text { Real time, } \\
\text { alarm monitoring }\end{array}$ \\
\hline 72 & GBR & 2 & 200 & $10^{-3}$ & $\begin{array}{c}\text { Real time, } \\
\text { live monitoring }\end{array}$ \\
\hline 73 & NGBR & 3 & 300 & $10^{-6}$ & $\begin{array}{c}\text { Delay tolerant, } \\
\text { metering }\end{array}$ \\
\hline \multicolumn{7}{|c|}{ * The priority is related to MTC traffic type } \\
\hline
\end{tabular}

these three types, as a base line in this paper, could be extended to provide precise differentiation if needed. The index values of the new QCIs are 71, 72, and 73, which are shown in Table IV and chosen from the spare QCI values.

In Table IV, the GBR resource type has been specified to the real time and delay sensitive MTC traffic, the NGBR resource type has been specified to the delay tolerant MTC traffic, the priority setting is related to the MTC traffic, in order to provide seamlessly integration manner with the standard QCIs assigned priority values. PDB and PLR are specified to fulfil the minimum NQoS required to provide acceptable levels to AQoS needs for MTC traffic.

\section{B. Proposed Network Architecture}

Fig. 2 shows the proposed network architecture for bridging the WSN devices with the EPS network. The integration is

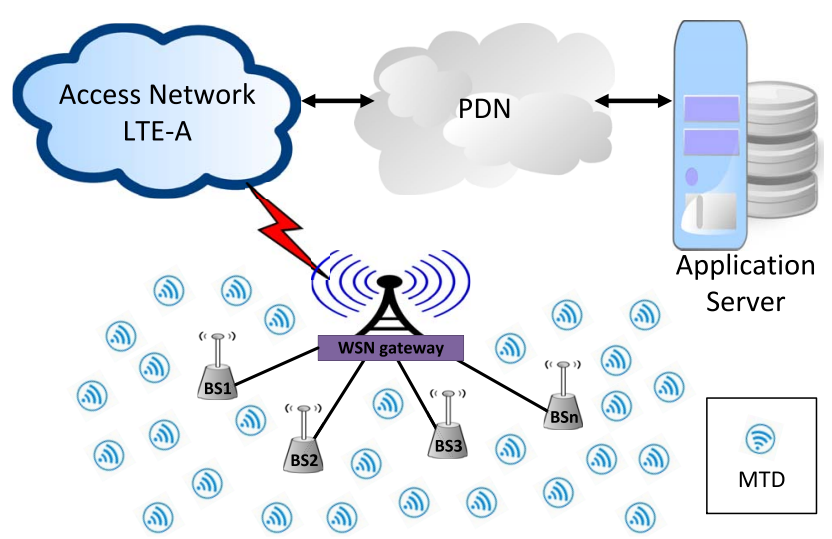

Fig. 2. Proposed network architecture.

achieved via a WSN gateway, which is in turn connected to the WSN base stations or coordinators.

The infrastructure of the proposed network is a combination of different WSNs that use different routing protocols, including IEEE 802.15.4 (ZigBee), IPv6 over low power wireless personal area networks, and the low-energy adaptive clustering hierarchy protocol (LEACH). Each WSN has a coordinator or base station that provides the harvested information to a WSN gateway. The gateway's design, implementation and its related used protocols are explained in detail in the following section.

\section{Access Network (WSN Gateway Design)}

WSNs are fundamental aspect for enabling MTC moving toward IoT technology in smart cities; they collect the data 


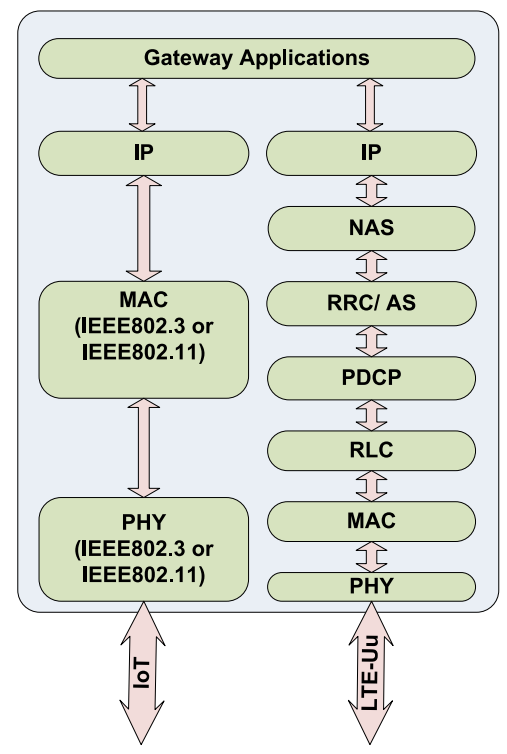

Fig. 3. WSN gateway protocol stack.

from the targeted areas and pass them through wireless communication. These networks can be connected to a higher level system (such as LTE operator networks) via a gateway, the main function of which is to provide connectivity and interaction between the WSN base station and the external PDN, transparently.

In the proposed scheme, the WSN gateway connects a mobile broadband network access node with WSN IoT infrastructure (as converged networks). In this paper, the methodology used to connect a massive WSN to a PDN via mobile broad network infrastructure is focused on the connectivity of the WSN traffic and QoS issues, as well as in consideration of QoE of real time applications users. The WSN gateway serves as a dual mode node and as a protocol converter between WSN base station and an E-UTRAN access node. An LTE-A Pro eNB access node is presented that introduces LTE-A as a mobile broadband network involved as a base line in this paper and for future NGN. The integration of multiple networks has two main types, tight and loose coupling techniques or topologies [30], with each type having its advantages and drawbacks from a QoS point of view. Regarding the loose coupling technique, the integration is offered by the WSN gateway, which provides dual mode connectivity between E-UTRAN and the WSNs. The protocol stack of a WSN gateway is shown in Fig. 3, where a protocol conversion is applied between the WSN coordinator and LTE-A traffic flows.

The control plane is assumed to be set up already among the $\mathrm{CN}$, eNB, and end devices. This includes the RAP, the NAS signaling, and the radio resource control (RRC). After completing the set up phase, the packet data convergence protocol (PDCP) applies the TFT packet filtering process and assigns bearers to its logical channel prioritization levels [31]. This process controls and divides the available network resources in the UL and DL directions among different bearers/SDFs over the entire EPS network [32]. These key features of controlling the QoS level of the initiated bearer between WSN gateway and PDN-GW are performed in PDCP layer in the WSN gateway for the UL direction and the GPRS tunneling protocol user plane layer in PDN-GW for the DL direction. The setting of the TFT filtering process, bearers identifier (ID), tunnel endpoint ID, and bearers IP address are instructed between PCRF and PDN-GW in the CN.

An efficient multiplexing algorithm for MTC traffic has been implemented between the WSN gateway and PDN-GW. MTC traffic is aggregated in the WSN gateway and then multiplexed together, which is carried out in the PDCP layer in the UL direction on the bearer level. The aggregation and multiplexing of different MTC traffic is based on newly defined QCI values to match the required QoS levels for MTC traffic flows during their transmission via the mobile broadband network. This WSN gateway is a low cost device; it multiplexes different MTC traffic flows on the bearer level without use of any extra resources at the radio interface and in the backhaul. Instead of requesting a new resource allocation in the UL direction from the eNB for an individual WSN node or base station, the requesting process is performed by the WSN gateway for a group of multiplexed service data flows originating from MTDs, based on the QoS level. This multiplexing is undertaken intelligently in the PDCP layer using TFT and packet filtering on PDCP service data unit (SDU). This comes from the IP layer, representing different MTD traffic flows and then produces a multiplexed PDCP packet data unit (PDU) to be mapped to the earlier initiated data radio bearers between the WSN gateway and PDN-GW. An overview of the proposed mechanism is shown in Fig. 4.

\section{Simulation Setup and Results}

\section{A. Simulation Setup}

The performance of the proposed network model is studied using the Riverbed Modeler version 18.5.1 [24] simulator from Riverbed Technologies Ltd. ${ }^{1}$ This version supports 3GPP standard and the modeler library offers a variety of protocol types and device models for supporting accurate simulation scenarios [33]. The modifications of the standard modeler models are carried out in order to implement the proposed simulation setup [24].

The designed E-UTRAN consists of one macro eNB, which is connected to EPC and PDN via an optical transport network comprising 7 WSN gateways and 30 active UEs randomly distributed within the coverage area of the eNB. The WSN gateways and UEs are positioned randomly in the coverage area of the eNB. Each WSN gateway is connected to one WSN base-station (coordinator) via an Ethernet connection using the IEEE 802.3 protocol, which is used to convey the harvested information toward the application server. The application servers are connected to the EPS network through the Internet backbone network. End device applications service request and call setup supports the use of session initiation protocol (SIP), which is configured among the UEs, WSN gateways, applications servers, and IP and multimedia subsystem (IMS) server. The simulated network contains profile definition, application

\footnotetext{
${ }^{1}$ Licensed Software offered by Brunel University London.
} 


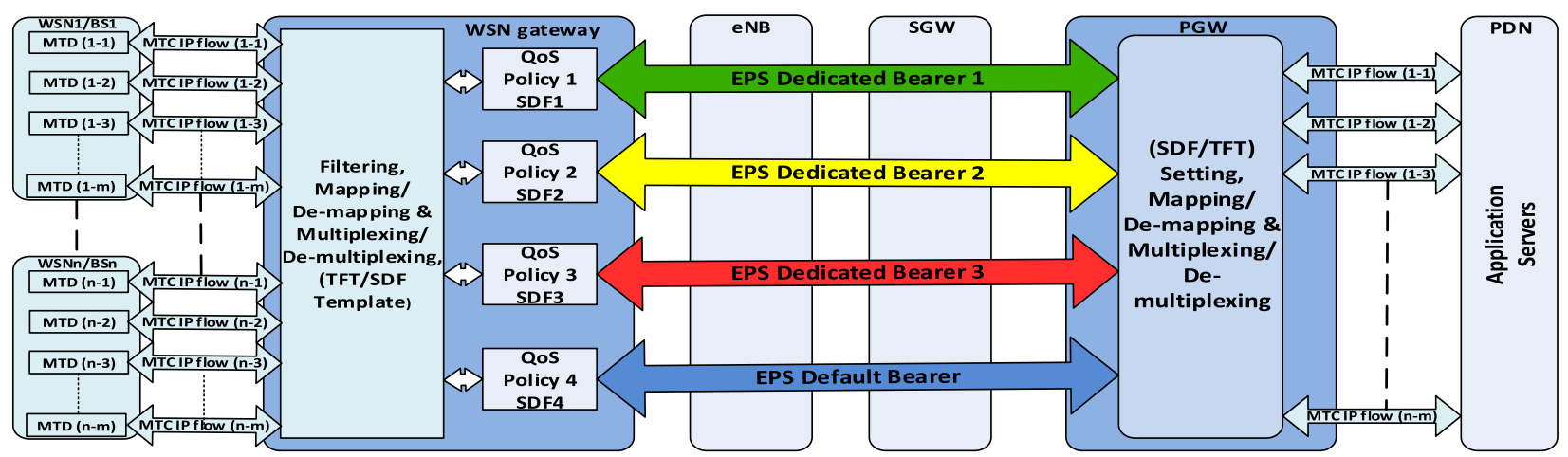

Fig. 4. Multiplexing process.

TABLE V

Simulation CONFIGURATION PARAMETERS

\begin{tabular}{|c|c|}
\hline Parameter & Setting \\
\hline No. of eNB & one single sector \\
\hline eNB coverage radius & $2000 \mathrm{~m}$ \\
\hline MAC Layer/ PSCH Scheduling Mode & $\begin{array}{l}\text { Link adaptation \& channel dependant scheduling } \\
\left.\text { (Max. } I_{M C S}=28, I_{T B S}=26\right)\end{array}$ \\
\hline IP Layer QoS (Scheduling Algorithm)/ Outside EPS rules and functions & Default (Best Effort) \\
\hline Path loss model, Fading Model and Shadow Fading Loss Model & UMa (ITU-R M2135) [34] \\
\hline UL Multipath model & LTE SCFDMA ITU Pedestrian A \\
\hline DL Multipath model & LTE OFDMA ITU Pedestrian A \\
\hline UE velocity & $0 \mathrm{~km} / \mathrm{sec}$ \\
\hline eNB, UE and WSN gateway receivers sensitivity & $-200 \mathrm{dBm}$ \\
\hline MIMO transmission technique & Spatial Multiplexing 2 Codewords 4 Layers \\
\hline UE and WSN gateway No. of receive antenna & 4 \\
\hline UE and WSN gateway No. of transmit antenna & 1 \\
\hline eNB No. of receive antenna & 4 \\
\hline eNB No. of transmit antenna & 4 \\
\hline Cyclic Prefix & Normal (7 symbols/slot) \\
\hline Duplexing Mode & FDD \\
\hline System BW & $20 \mathrm{MHz}$ \\
\hline PUCCH, PDCCH \& PHICH configurations & Modeler default values \\
\hline UL carrier frequency (operating band 1) [16] & $1920 \mathrm{MHz}$ \\
\hline DL carrier frequency (operating band 1) [16] & $2110 \mathrm{MHz}$ \\
\hline UE and WSN gateway Antennas Gain & $-1 \mathrm{dBi}$ \\
\hline eNB Antenna Gain & $15 \mathrm{dBi}$ \\
\hline eNB, UE and WSN gateway maximum transmission power & $27 \mathrm{dBm}$ \\
\hline Modulation and Coding Scheme (MCS) & Adaptive \\
\hline No. of TTI & 140000 \\
\hline eNB, UE and WSN gateway PDCP Layer Max. SDU & 1500 Bytes \\
\hline PDCP layer Robust Header Compression Ratio (RoHC) & $\begin{array}{l}\text { UDP/IP:- uniform }(0.5714,0.7143) \\
\text { TCP/IP:- uniform }(0.1,0.4) \\
\text { RTP/UDP/IP:- uniform }(0.1,0.15)\end{array}$ \\
\hline UL/DL HARQ maximum retransmission & 3 \\
\hline
\end{tabular}

definition, LTE configuration, and mobility management entities. Furthermore, the path-loss, fading, and shadow models used in the wireless links are the Urban Macro-cell ITU-R M2135 model [34]. $20 \mathrm{MHz}$ has been set in the EPS RAN part with maximum radius of coverage of $2 \mathrm{~km}$, in order to provide a realistic scenario for the wireless part of the simulated network. The typical E-UTRAN parameter settings are listed in Table V. Ten UEs initiate real time voice call by using the VoLTE service, where five of them are the voice callers and the other five are voice called. This service is set to use high data rate codec (G.711) with voice payload frame size of $10 \mathrm{~ms}$ and 80 Bytes per frame [35], this is going to produce one digitized voice payload frame every $10 \mathrm{~ms}$ with $64 \mathrm{~kb} / \mathrm{s}$ rate, which is the voice application layer PDU. The consequential physical layer VoLTE PDU rate will be $96 \mathrm{~kb} / \mathrm{s}$, including all the protocols overhead with RoHC [24]. The VoLTE bearer traffic stream bandwidth in the physical layer matches and occupies the maximum GBR bearer setting, that was designed for the VoLTE service, and this will produce a full utilization to the voice bearer bandwidth and this verifies that G.711 is a resource hungry codec. The size of $10 \mathrm{~ms}$ voice frame was chosen to support the QoE estimation process of VoLTE service with the required parameters. Another ten UEs initiate real time video call by using a video conferencing service, 
TABLE VI

H2H SERVICE AND APPLiCATION CHARACTERISTICS

\begin{tabular}{|l|l|l|}
\hline Service/ Application & Application Layers Parameter & \multicolumn{1}{|c|}{ Setting } \\
\hline \multirow{4}{*}{ Voice } & Encoder Scheme & G.711 \\
& Frames per Packet & 1 \\
& Frame Size & $10 \mathrm{msec}$ \\
& Compression/ Decompression Delay & $20 \mathrm{msec}$ \\
& ToS/ DSCP & Interactive Voice/ CS6 \\
\hline \multirow{3}{*}{ Video Conferencing } & Frame Interarrival Information & 10 frames/sec \\
& Frame Size Information (Bytes) & $128 x 120$ pixels \\
& ToS/ DSCP & Streaming Multimedia/ CS4 \\
\hline \multirow{5}{*}{ FTP } & Command Mix (Get/Total) & $100 \%$ \\
& Inter Request Time & Uniform, min. 27 sec, max. 33 sec \\
& File Size & Constant, 500 Bytes \\
& ToS/ DSCP & Standard/ CS2 \\
\hline \multirow{3}{*}{ HTTP } & Specification & HTTP 1.1 \\
& Page Interarrival Time & Exponential, 60 sec \\
& ToS/ DSCP & Best Effort/ CS0 \\
\hline
\end{tabular}

TABLE VII

MTD'S TRAFFIC CHARACTERISTICS

\begin{tabular}{|l|c|c|c|}
\hline \multicolumn{1}{|c|}{ Service / Application } & ToS/ DSCP & IP Layer Packet Size & IP Layer Packet Rate \\
\hline Alarm & Excellent Effort / CS3 & \multirow{2}{*}{512 Bytes } & \multirow{2}{*}{ 4 Packets/sec } \\
\cline { 1 - 2 } Live Monitoring \& Remote Monitoring & Streaming Multimedia / CS4 & \\
\hline Metering & Background / CS1 & \\
\hline
\end{tabular}

where five of them are the callers and the rest are called. Regarding the remaining ten UEs, 5 uses the FTP application to communicate with remote server 1 and the other five utilizes the HTTP application to communicate with remote server 2. $\mathrm{The} \mathrm{H} 2 \mathrm{H}$ services and application characteristics are listed in Table VI.

The seven WSN gateways receive traffic generated from the MTDs, which is routed via the WSN coordinators. Each MTD is programmed to generate its traffic as a time based event activation. The rate of activation of the MTDs at each WSN platform is 5 MTD per second in an accumulating fashion, starting at $135 \mathrm{~s}$ and continuing until the end of the simulation. By the end of the simulation, each base station and WSN gateway will have received and routed the traffic of 500 MTDs. The metering MTDs have an $80 \%$ share of the generated traffic, while alarm and live monitoring MTDs have $10 \%$ for each of them. The MTDs generated traffic is proposed as being created from one of following WSN protocols: constant bit rate routing protocol, LEACH or ZigBee, and the MTDs traffic characteristics are listed in Table VII, which are related to MTDs traffic settings in [36]. Moreover, the bearer configuration settings in the Riverbed modeler are listed in Table VIII.

Two identical and separated simulation scenarios were build in a single Riverbed modeler project in order to facilitate the scenarios run sequencing process as well as the comparisons procedures of the measurements. The first one was pertained to considering MTC traffic as a best effort traffic flow, whereas the second was about using the proposed policy for traffic flow management. Each scenario was run for 30 times sequentially with randomly chosen seeds [37], [38], since producing data from a single execution cannot give a correct picture of the performance of the framework [39]. Furthermore, these scenarios run replications were set with multiseed value settings of the random number initializer, in order to get an outcome with a confidence interval of $95 \%$ as an estimated validity of the results, which is the resultant from above mentioned replications count [24], [37], [39]. Specifically, this approach is designed in Riverbed Modeler software to support confidence estimation for scalar data reporting [24], [40], which is collected in multiseed parametric runs. The confidence interval marker is not shown in the results figures, in order to emphasis and highlights the assessment concerning the aforementioned two scenarios. The designed baseline EPS network model is shown in Fig. 5.

\section{B. Simulation Results}

Two Riverbed Modeler simulation scenarios with the proposed WSN gateway have been conducted in order to evaluate and validate the performance of the suggested system. For both scenarios, the simulation run time was set at $240 \mathrm{~s}$. This time was specified to match the estimated traffic capacity of the PUSCH, with respect to the number of the attached devices and their aggregated UL traffic loads rates at the eNB. Furthermore, to inspect how the conducted simulation of proposed traffic management policy on the bearers level provoked at the physical layer level. In this regard PUSCH utilization and throughput, once the bearers are starting the competition when there is a scarcity in the network resources. UL capacity mainly depends on the proposed physical layer structure parameters, PUCCH and random access configurations as shown in Table V of eNB, UEs, and WSN gateways. When the UL traffic loads reach the estimated UL capacity, it 
TABLE VIII

BEARER CONFIGURATION

\begin{tabular}{|c|c|c|c|c|c|}
\hline QCI & ARP & $\begin{array}{c}\text { Resource } \\
\text { Type }\end{array}$ & $\begin{array}{c}\text { Max. \& GBR } \\
(\mathrm{kbps})\end{array}$ & $\begin{array}{c}\text { TFT Packet Filters } \\
\text { DSCP value }\end{array}$ & $\begin{array}{c}\text { Service/ } \\
\text { Application }\end{array}$ \\
\hline 1 & 1 & GBR & 96 & CS6 & Video Conferencing \\
\hline 2 & 2 & GBR & 384 & CS4 & FTP \\
\hline 6 & 6 & NGBR & 384 & CS2 & HTTP \\
\hline 7 & 7 & NGBR & 384 & CS0 & Live Monitoring \\
\hline 71 & 3 & GBR & 384 & CS3 & Metering \\
\hline 72 & 4 & GBR & 384 & CS4 & SIP Protocol IMS Signalling \\
\hline 73 & 8 & NGBR & 384 & CS1 & Low Priority Application \& Undefined DSCP \\
\hline 5 & 5 & NGBR & 384 & Modeler default setting & BE \\
\hline 9 & 10 & NGBR & 384 & &
\end{tabular}
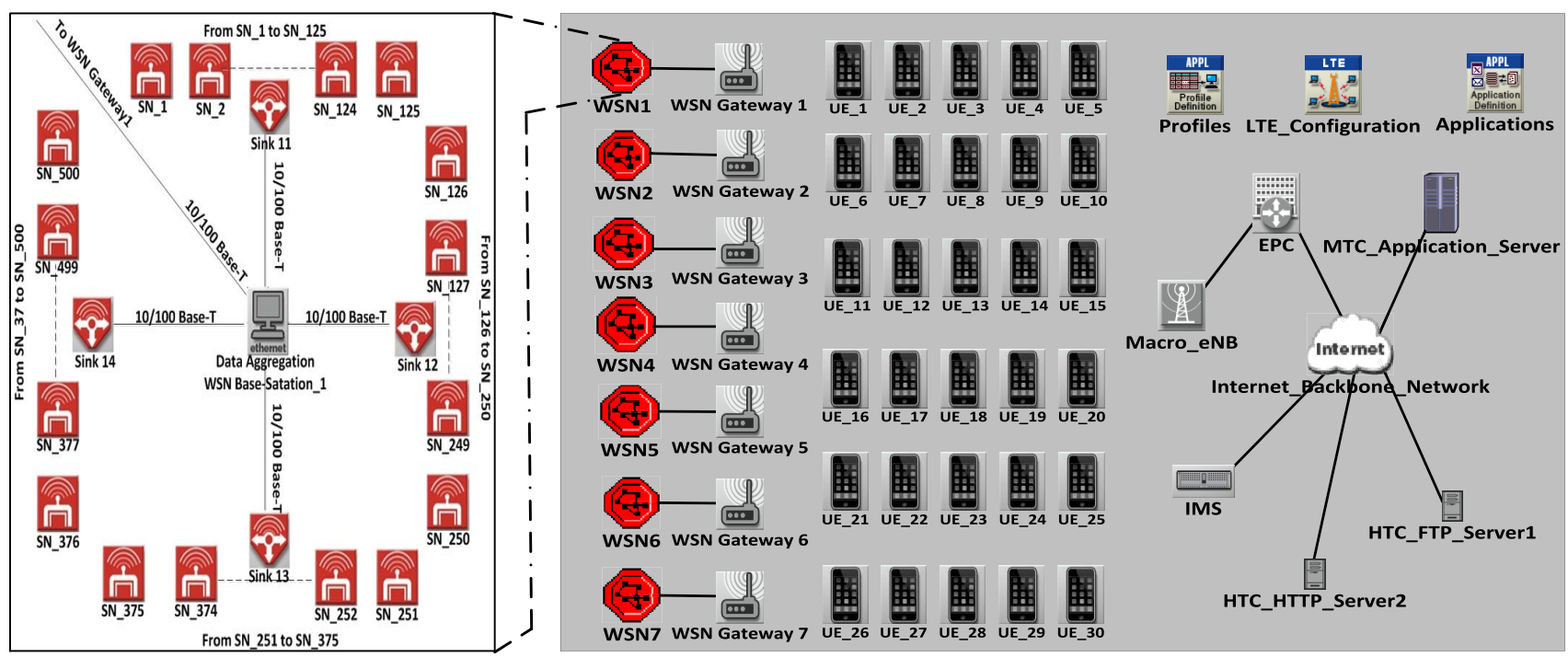

Fig. 5. Riverbed modeler network setup.

will be shared among the number of attached devices to the eNB and their UL traffic loads. The PUSCH capacity represents the bottleneck in the LTE-A networks [41]. According to [24], [26], and [42], the estimated data plane PUSCH traffic capacity is $73.05 \mathrm{Mb} / \mathrm{s}$ per TTI. Whereas, the planned maximum aggregated data plane PUSCH traffic loads, of the services and applications in the simulation scenarios, they will achieve $70.7 \mathrm{Mb} / \mathrm{s}$ per TTI at $235 \mathrm{~s}$. The reminder $2.35 \mathrm{Mb} / \mathrm{s}$ per TTI are left for IMS, SIP, SDP, RTP, RTCP, and other application layers control signaling traffics, such as transport, session, presentation, and application layers. Since the latter protocols, services, applications, and layers traffic loads are measures of data plane traffic loads apart from LTE network control plane signaling overheads, such as NAS and RRC [14].

To provide an acceptable estimation of the long term performance measure for the proposed framework. The generated traffic, from applications used by any node in the simulation models, is only created when the application is active. The default setting of Riverded Modeler requires a network warm-up time at the beginning of the simulation experiment [24], which is typically set to be $100 \mathrm{~s}$ [43]. This time is important in any network simulation model run for the first time from an empty scenario to allow queues and other simulated network parameters reach a steady-state running conditions. In addition, it allows other devices such as routers and servers in the simulated scenario to initialize. Therefore, $100 \mathrm{~s}$ warm-up time provides a sufficient amount of time for all the network protocols to initialize and converge to their stable states [37], [43]. The RAPs and attaching processes of the HTDs and WSN gateways to the EPS were done between 100 and $105 \mathrm{~s}$ of the simulation run time, on the random basis with different time instances for each device. The HTC services request and the applications starting times were set within 105 to $110 \mathrm{~s}$ of the simulation run time. The MTC services and applications traffic flows starting and initialization times were set at $135 \mathrm{~s}$ as mentioned in Section IV-A.

Fig. 6 shows the total records of the PUSCH utilization percentage, aggregated PUSCH throughput, and $\mathrm{PDCCH}$ utilization percentage of the eNB, respectively, during the simulation runs. In Fig. 6(a) and (b), the proposed model gave a better utilization percentage and a good throughput, respectively. The default model utilized $100 \%$ of PUSCH at 174 s, with maximum aggregated PUSCH throughput of $37 \mathrm{Mb} / \mathrm{s}$ per TTI. While the proposed model achieved $100 \%$ PUSCH utilization at $228 \mathrm{~s}$, with maximum aggregated PUSCH throughput of 64 $\mathrm{Mb} / \mathrm{s}$ per TTI. At $174 \mathrm{~s}$ there were 1400 active MTDs, while at $228 \mathrm{~s}$ were 3290 active MTDs. The PDCCH reaches full capacity utilization in the default model at $228 \mathrm{~s}$ of simulation run time, while in the proposed model performs $95 \%$ utilization at congestion condition, as shown in Fig. 6(c). It is clear 


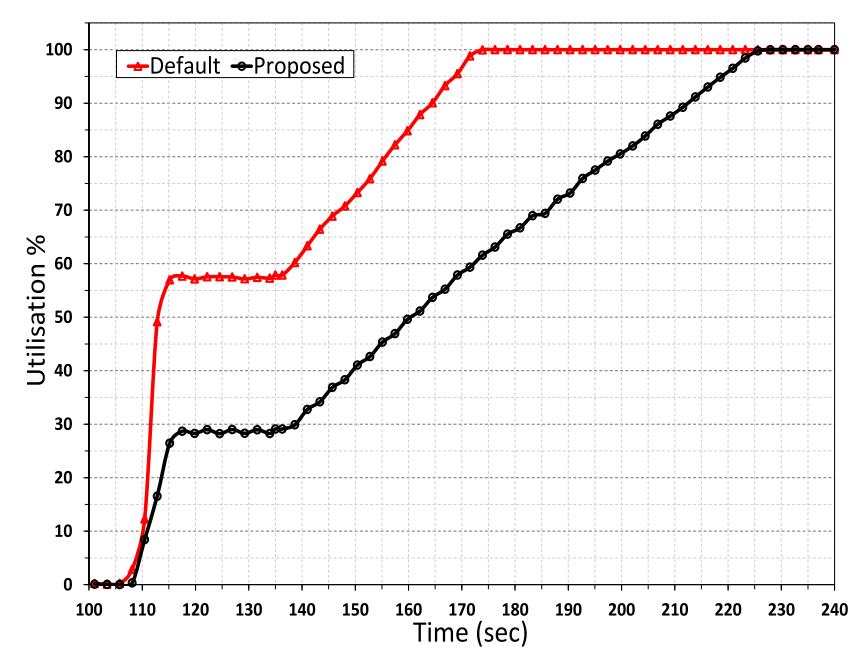

(a)

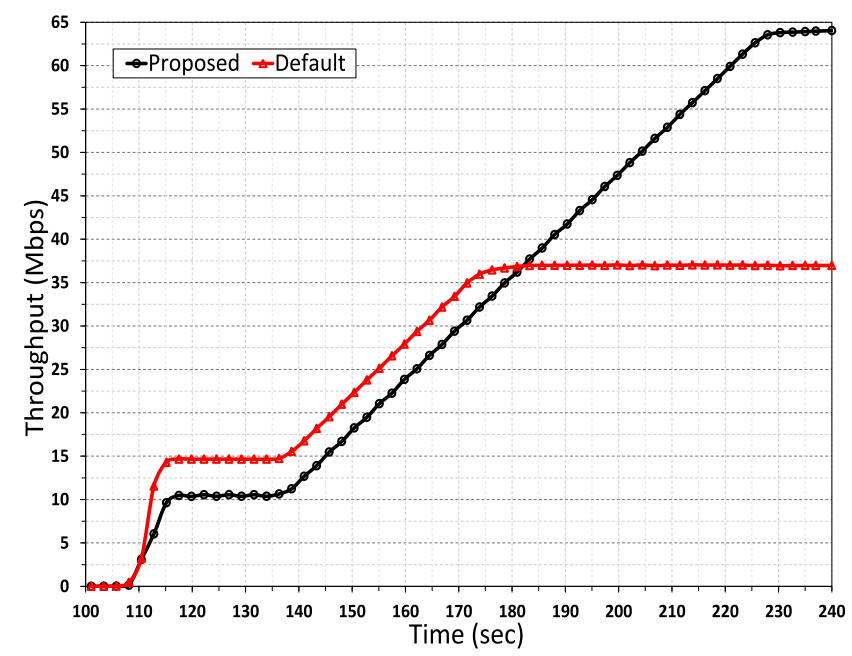

(b)

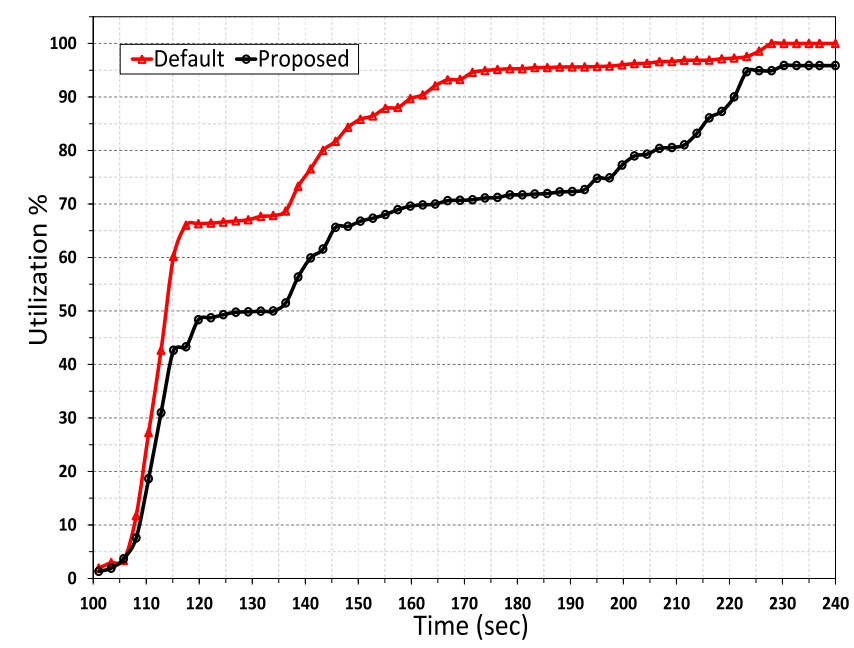

(c)

Fig. 6. eNB performance. (a) PUSCH utilization. (b) PUSCH throughput. (c) PDCCH utilization.

from these physical layer KPIs the eNB has got an improvement in PUSCH utilization, PUSCH throughput, and PDCCH utilization during congestion, fully or over utilization links,

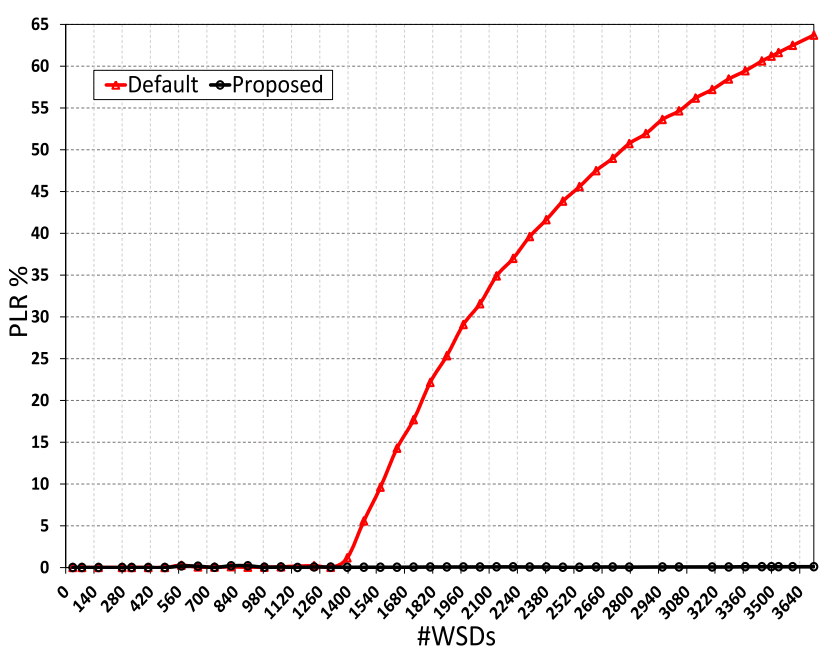

(a)

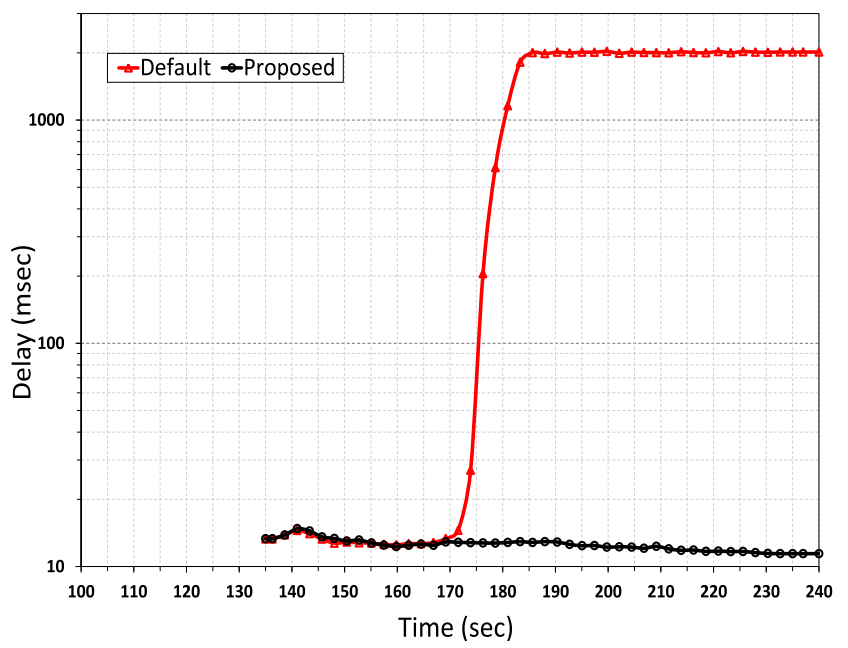

(b)

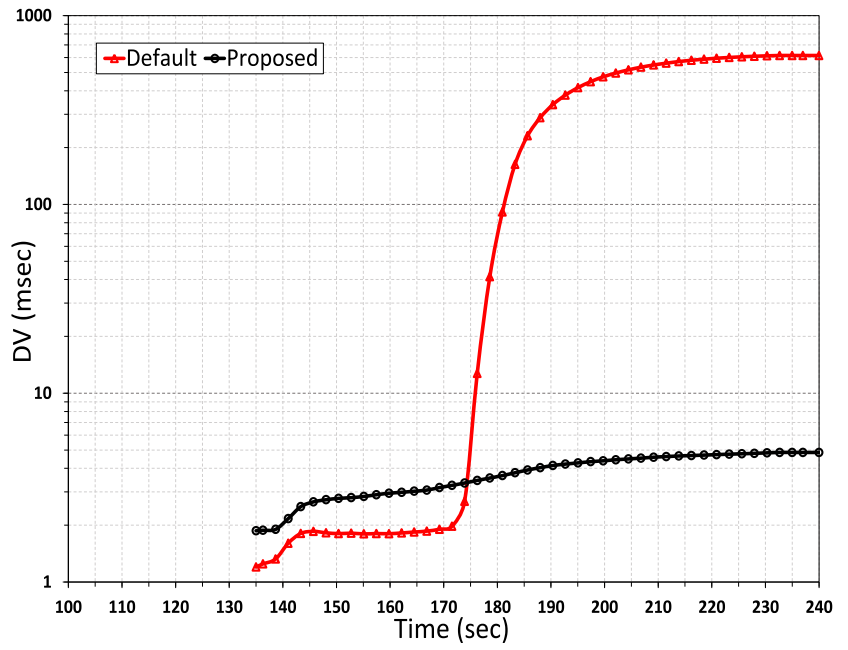

(c)

Fig. 7. Simulation results for MTC traffic. (a) PLR of MTC's traffic. (b) E2E packet delay of MTC's traffic. (c) PDV of MTC's traffic.

besides normal traffic conditions. The E2E NQoS performance measurements will show us the impact of the proposed traffic flow management policy on the HTCs and MTC's traffic flows, as demonstrated in the next paragraphs. 


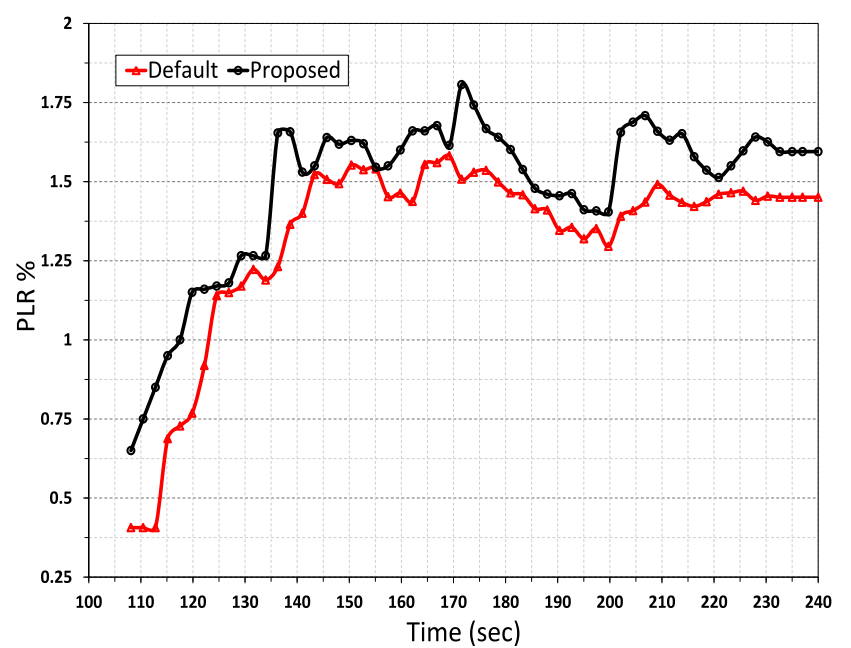

(a)

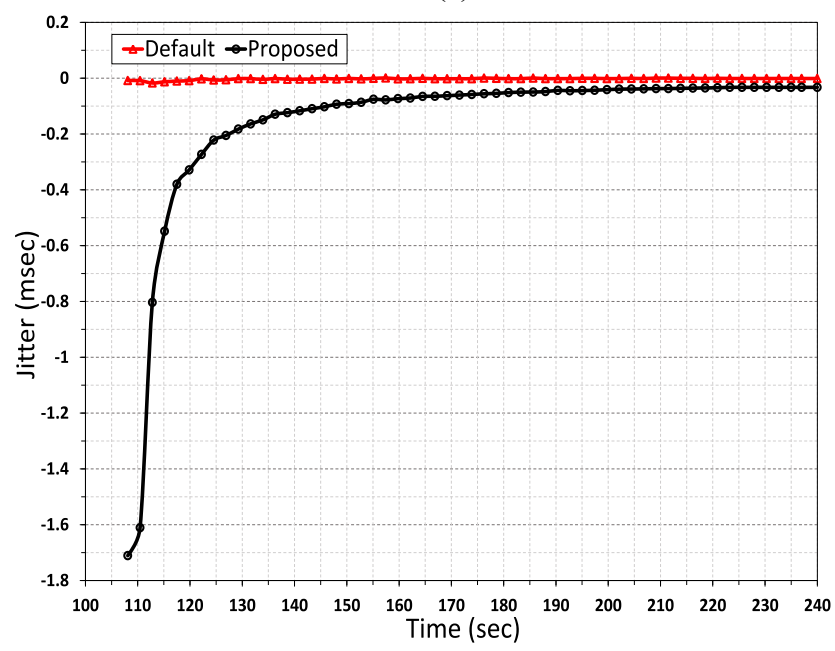

(c)

Fig. 8. Simulation results for VoLET service. (a) PLR of the VoLTE service. service. (d) MOS of the VoLTE service.

Fig. 7 demonstrates the results for the MTC traffic. Fig. 7(a) represents the percentage of the PLR of the received traffic from all active MTDs with respect to their number in the whole network for the default and proposed models. This figure shows a comparison of PLR for these two models and it clearly shows a significant reduction in the packet loss levels for that proposed.

Fig. 7(b) demonstrates one way E2E packet delay between one of the WSN coordinators and the MTC application server at the IP layer, with respect to the simulation run time. The default network model E2E packet delay increased as the traffic generated from newly activated MTDs, they were joined the network cumulatively, the delay was $100 \mathrm{~ms}$ at $175 \mathrm{~s}$ of simulation run time, then it was increased dramatically after that time and reached $2016 \mathrm{~ms}$. While the proposed network model gave a significant reduction in the received E2E packet delay levels, where the E2E packet delay range between 11.5 and $15 \mathrm{~ms}$.

Fig. 7(c) shows the average IP packet E2E delay variation in the default and proposed models. This metric was measured

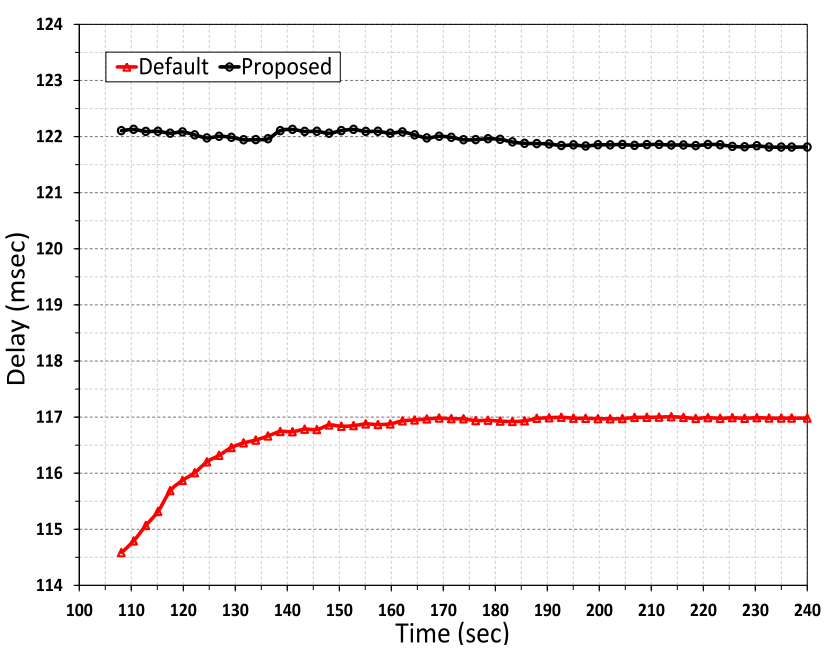

(b)

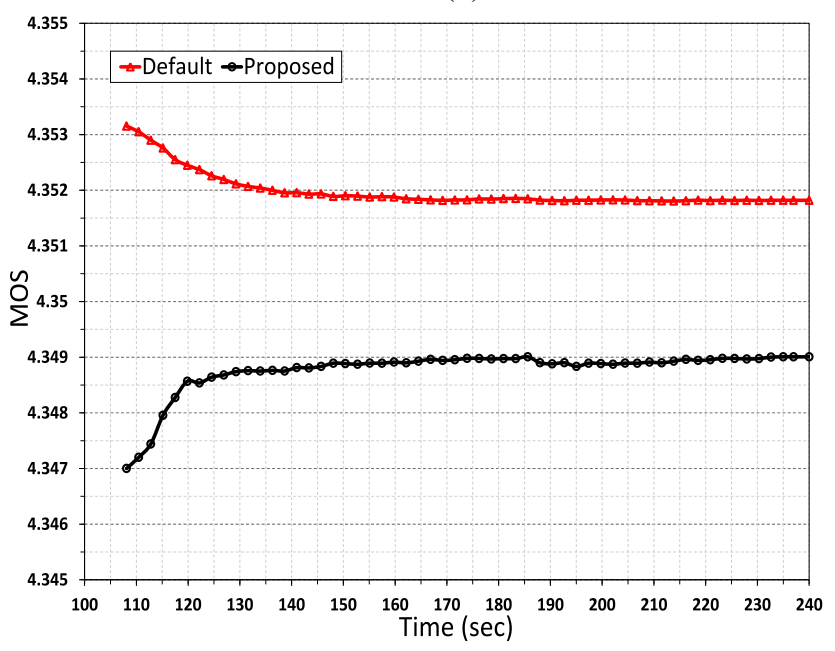

(d)

(b) E2E packet delay of the VoLTE service. (c) Packet jitter for the VoLTE

in the IP layers of one of the WSN base station (labeled: WSN2) and the MTC application server. The default model gave a maximum average IP E2E delay variation of $615 \mathrm{~ms}$, while for the proposed model, this metric was between 1.87 and $4.856 \mathrm{~ms}$. The delay variation is very important, since it affects the monetisation and in sequence delivery of data streams to the transport layer on the receiver side.

The simulation results of the VoLTE service are shown in Fig. 8, which includes PLR as one of the important metrics of NQoS of VoLTE. It measures the error rate in the packet flow stream, which is forwarded to the voice application by the transport layer. Fig. 8(a) shows the average E2E PLR in the packets between two calling parties. This figure shows an increase in the PLR value with respect to simulation run time in the default and proposed models. The proposed models PLR was more than the default one, with the maximum values being $1.81 \%$ and $1.61 \%$, respectively.

The PLR per voice bearer in both scenarios remained lower than the maximum allowable limit, which is $1 \%$ for the voice application bearer as presented in Table I [20]. The measured 
value represents the total accumulated E2E PLR in the UL and DL directions, while the maximum defined voice bearer PLR limit represents a one direction PLR. Therefore, the average of the maximum achieved E2E PLR per voice bearer (UL and DL voice bearers) of the proposed model is $0.905 \%$, whereas for the default model is $0.805 \%$, which is a good level, since most of the up-to-date voice codecs perform well up to $1 \%$ PLR [44]. The irregular variation of the PLR during the simulation run time was due to the radio interface environment variation (path loss and fading effects in the UMa model) between eNB and the calling parties as well as the increased traffic loads, during the simulation runs. The consequence of increased traffic loads was throttling the UL, which created congestion in the PUSCH. VoLTE service packet delay is called mouth to ear delay, which is one of the most important factors to be considered when dealing with the QoS of real time voice service in PS, such as the VoLTE service in our network model. Fig. 8(b) shows the average E2E packet delay of the VoLTE in the default and proposed network models.

The default model average E2E packet delay started at $114.58 \mathrm{~ms}$ and reached $116.9 \mathrm{~ms}$ at $150 \mathrm{~s}$ of the simulation run time, then remaining at this value until the end of the simulation run. Whereas the proposed network model kept its average E2E packet delay at $122 \pm 0.1 \mathrm{~ms}$ from the start of the VoLTE service traffic flow until the end of the simulation run. This measure was taken as E2E, which means it was in the UL/DL directions. Obviously, there was a little degradation in this NQoS, because the proposed model gave higher delay values (about $7 \mathrm{~ms}$ ) than the default model. These values of average E2E delay in the default and proposed models would still fulfil the 3GPP and the ITU-R standardizations as well as the requirements for VoLTE service E2E packet delay, where the maximum allowable one way packet delay is $150 \mathrm{~ms}$ in order to accomplish high QoE [25], [45], [46].

Packet jitter is one of the most important QoS metrics of real time services. Fig. 8(c) shows the average jitter of the VoLTE service in the proposed and default models. In this figure, the default network model gave a better packet jitter performance than the proposed network model. The average packet jitter values of the default model were between -0.00844 and $-0.000974 \mathrm{~ms}$ for the received packets of the VoLTE service traffic up until the end of simulation run time. Whilst the proposed model's average packet jitter value for the VoLTE service traffic flow started at $-1.7 \mathrm{~ms}$ and by the end of the simulation it had decreased to $-0.0326 \mathrm{~ms}$. Clearly, there is degradation in this NQoS metric, for a consequence of the increased average packet jitter will be an increase in the time delay of the received packets in the de-jitter buffer [47]. The value of the average packet jitter in the proposed model was reduced. The ITU-R has recommended $25 \mathrm{~ms}$ jitter as an acceptable value for the delay variation [48].

The users QoE levels of real time voice call are a measure of users satisfaction levels when engaging with the VoLTE service. The MOS is a well-known metric to measure QoE by using its E-Model, according to ITU-T G107 [49]. This QoE metric can be reported as sampled, interval or cumulative metrics, using a modified instantaneous E-Model [50], [51]. MOS statistics are collected using instantaneous E-Model, it was set in the simulation models to report MOS value as a sample mean of 100 MOS instantaneous sampled statistics every $2 \mathrm{~s}$, which is called bucket mode [24]. Each one of the 100 MOS instantaneous sample statistic is collected within $20 \mathrm{~ms}$ time interval. Whereas, the voice codec frame size is set to treat 1 digitized voice frame every $10 \mathrm{~ms}$, meaning that the voice traffic has an interarrival time of $10 \mathrm{~ms}$. Therefore, the MOS instantaneous sampled statistics analysis relies on 2 current and 2 previous digitized voice frames QoS metrics, such as received voice application, frame delay and error rate [51], [52]. Fig. 8(d) shows the MOS of the default and the proposed network models. The default model started with MOS of 4.3532 then dropped to 4.3518 , whereas the proposed network model started at MOS of 4.347 then increased to 4.349. Moreover, the levels of QoE represented by the MOS value, maintained within good acceptable levels during the whole voice call time for both models.

A video conferencing service over LTE QoS parameters represented by PLR, packet delay, and packet jitter should be assured at certain levels in order to maintain a good QoE. Fig. 9(a) demonstrates the PLR for the default baseline and proposed network models with respect to the simulation run time. This QoS metric was measured in the received packet stream forwarded by the transport layer to the video conferencing application. The default model average PLR at the start of traffic flow at $108 \mathrm{~s}$ was $4.231 \%$ and this increased to $92.8 \%$ at the end of the simulation. Whilst the proposed network model was started with a PLR of $0.846 \%$, which then increased to $8.3 \%$ at the end of the simulation.

Fig. 9(b) shows the average E2E packet delay of the video conferencing service packet stream forwarded to the application layer from the transport layer at the destination point. In this figure, the QoS metric is represented by the average E2E packet delay for the default and proposed network models. The measurement starts from $108 \mathrm{~s}$ of simulation run time, when the video conferencing service starts its traffic flow, until its end. The proposed network model gave a better average packet delay performance than the default one during the whole period of the simulation run, especially at the end of simulation, which represents the heavy load traffic period. In this part, the proposed network model gave a better packet delay performance than the default one, since the maximum average packet delay in the proposed network model was started with $24.9 \mathrm{~ms}$ and ended with $36.5 \mathrm{~ms}$. Whereas, for the default network model was kept on $35 \mathrm{~ms}$, then at $228 \mathrm{~s}$ it was shot up and reached $256.5 \mathrm{~ms}$ at the end of simulation runs.

Another important QoS factor that has an impact on the interactive real time video conferencing service's $\mathrm{QoE}$, is the PDV. Fig. 9(c) shows the average PDV (APDV) with respect to the simulation run time of the video conferencing service for the received packets stream, which was forwarded from the transport layer toward the application layer at the called end device. This APDV was measured between the two video calling parties. In Fig. 9(c), it can be seen that the proposed network gave a higher APDV between 0.144 and $0.167 \mathrm{~ms}$ up to the end of the simulation run time. However, the default network model was started with $0.2458 \mathrm{~ms}$ and then, it gave 
a lower APDV of $0.2228 \mathrm{~ms}$. Furthermore, at point $227 \mathrm{~s}$ of simulation run time, the APDV increased rapidly and reached $1.457 \mathrm{~ms}$ by the end of the simulation. It is clear that the default models APDV increased rapidly, when the network was under a heavy traffic load, which could be a serious issue on the receiver side for real time playback video application [47]. The improvement of the video NQoS metrics in the proposed model over the default model is related to the GBR video bearer setting and allocation, such as QCI and ARP. It is also related to the use of the proposed traffic management policy, which is set as fixed share represented by $\alpha$ function (as listed in Table III). Its impact was clear when the eNBs UL has been fully utilized by HTC and MTC traffics. In the default model the video bearers NQoS metrics, such as PLR, packet delay, and PDV was kept within a specific range. However, at $228 \mathrm{~s}$ the capacity of PDCCH utilization in the default model reaches $100 \%$ as shown in Fig. 6(c). All traffics now compete for the PDCCH space. Therefore, the aforementioned NQoS metrics in default model it were shot up rapidly at the end of simulation, while in the proposed model it were kept within acceptable levels, as shown in Figs. 7-9.

Fig. 10 shows the results obtained of the FTP and HTTP applications in terms of E2E packet delay, PDV, and TCP retransmission metrics. FTP NQoS metrics has been inspected in the FTP application server as a destination point at the transport layer. The FTP as a nonreal time service and it uses the TCP retransmission mechanism between the source and destination points. Fig. 10(a) shows the average E2E packet delay for all packets with respect to the simulation run time, which was sent from the UEs that using FTP application, for the default and proposed network models. The proposed network model gave a higher packet delay in comparison to the default network model. Specifically, the proposed network models average packet delay was between 13.74 and $14.04 \mathrm{~ms}$, whereas that for the default model was between 12.76 and $13.47 \mathrm{~ms}$.

E2E delay variation represents another important QoS factor for the FTP application and Fig. 10(b) shows the average for packets at the IP layer between one of the UEs and the FTP application server. It is clear that the proposed network models delay variation was higher than the default network model. The maximum average for the former was $9 \mathrm{~ms}$, while that for the latter was $7.5 \mathrm{~ms}$. As was mentioned before, the FTP application will use the TCP retransmission request mechanism, which could be reflected as lost or outdated packets at the transport layer.

Fig. 10(c) demonstrates the average TCP retransmission count with respect to simulation run time for the default and proposed network models. It shows that, with the default model, no retransmission occurred during the FTP application traffic flow period. While for the proposed network model it did happen, which means that either there was a network congestion or a loss of packets received in the transport layer.

HTTP service QoS factors were checked at the HTTP application server in the transport and IP layers. This is a nonreal time service, which uses the TCP retransmission mechanism between the user and the application server. Fig. 10(d) shows

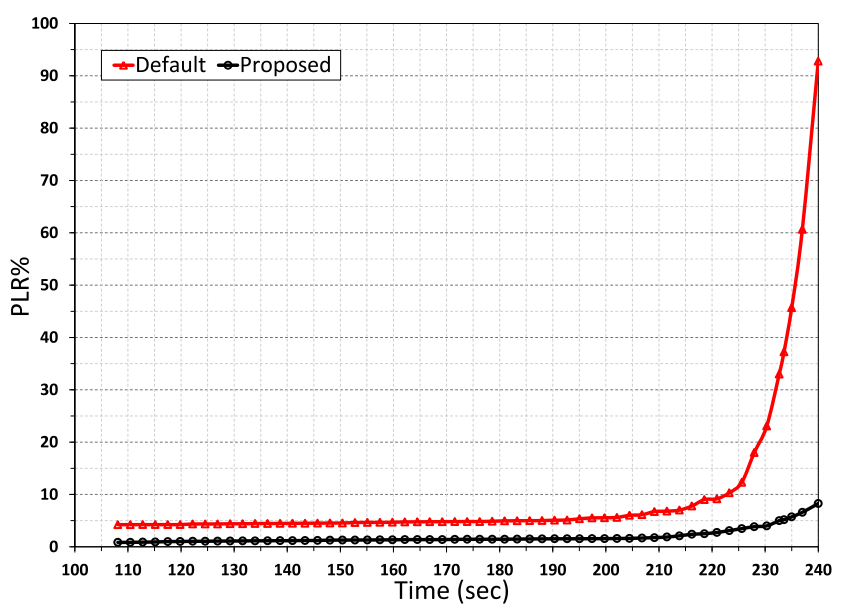

(a)

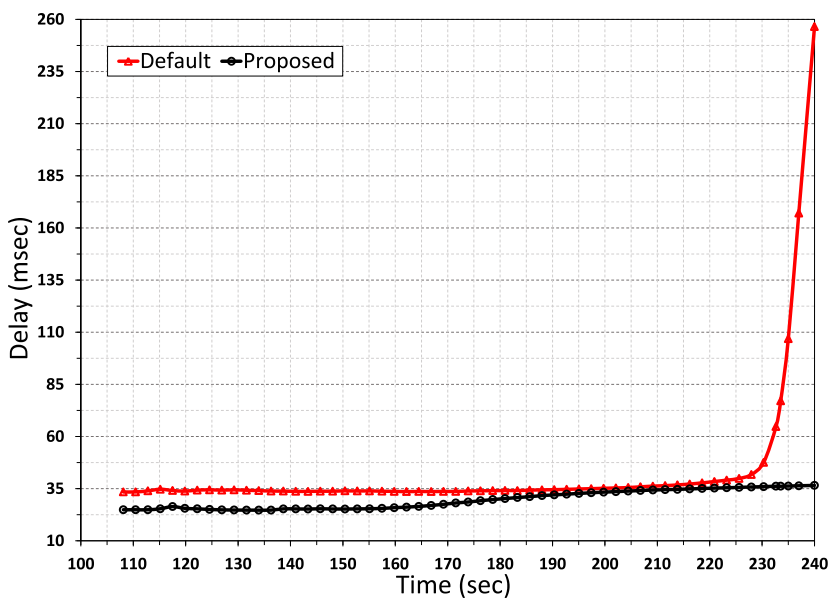

(b)

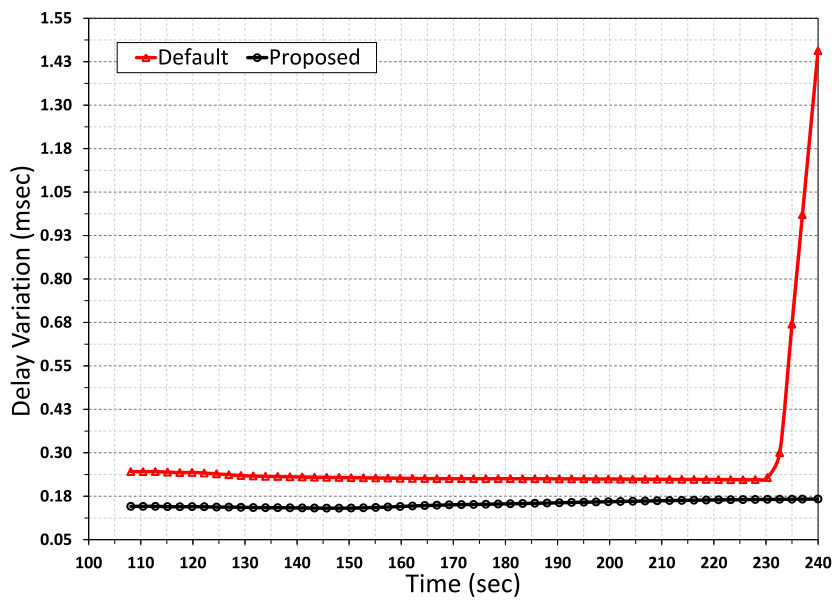

(c)

Fig. 9. Simulation results for video conferencing service. (a) PLR of the video conferencing service. (b) E2E packet delay of the video conferencing service. (c) PDV of the video conferencing service.

the average E2E packet delay for all received packets at the transport layer with respect to the simulation run time, which has been sent from the UEs that use the HTTP service application, for the default and proposed network models. The proposed network model gave almost a constant average delay, whilst the default network model did not. 


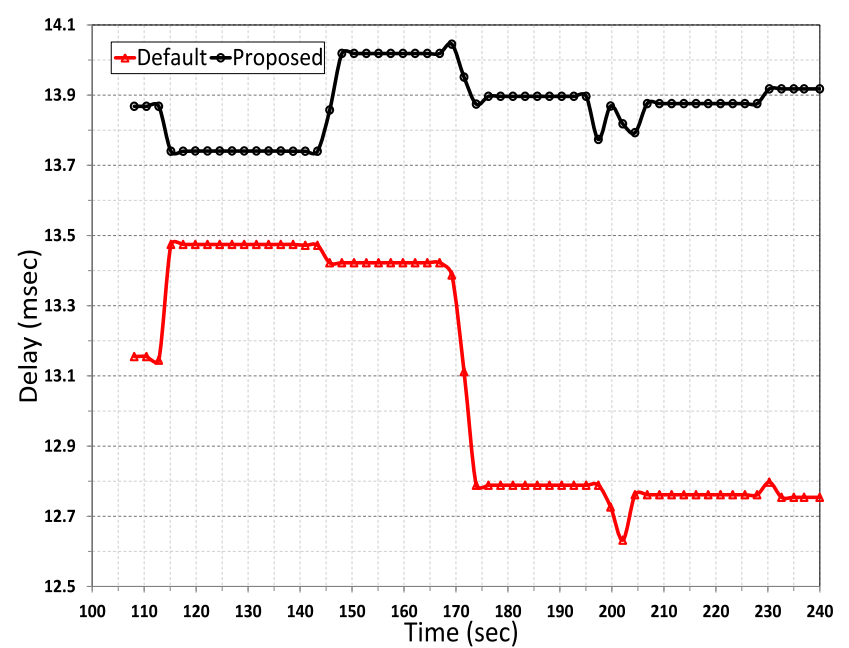

(a)

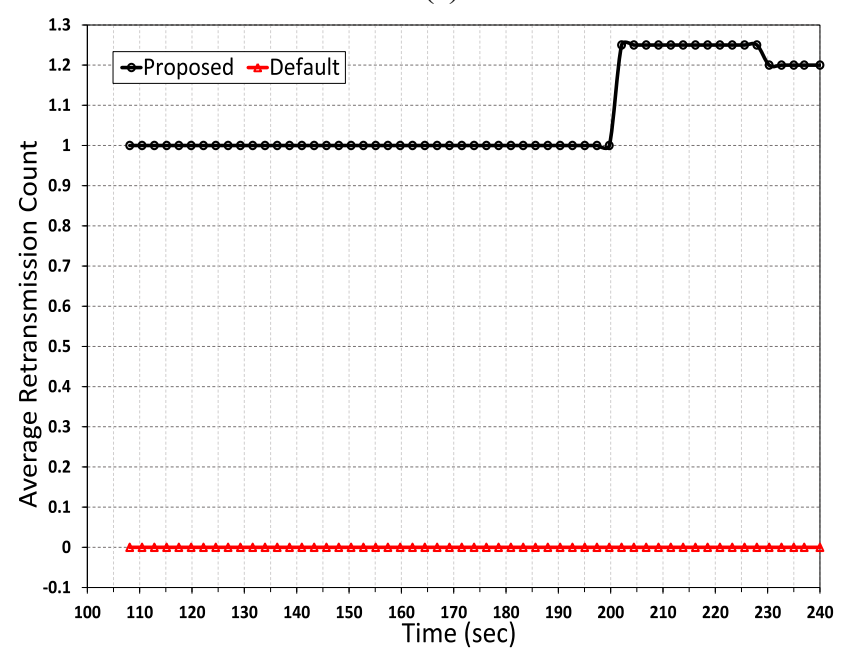

(c)

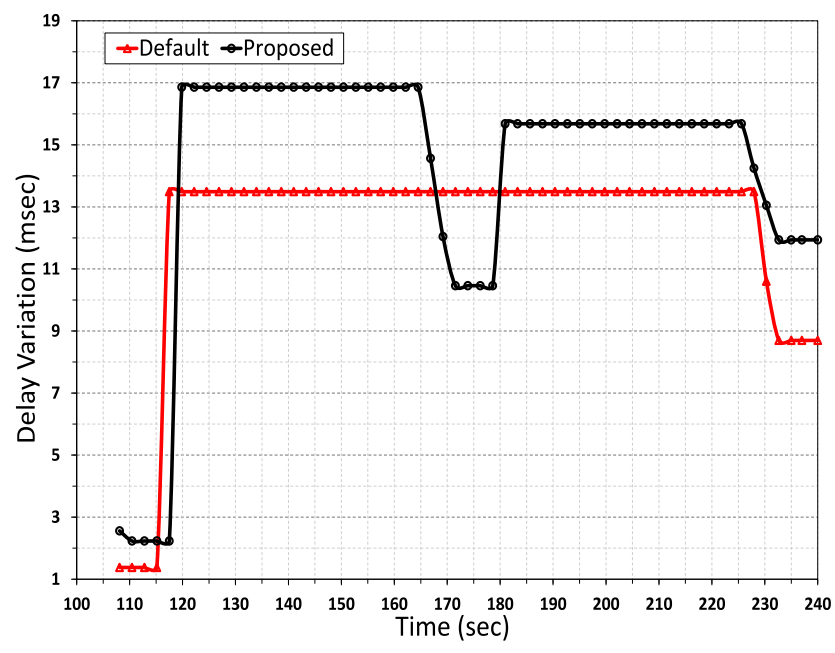

(e)

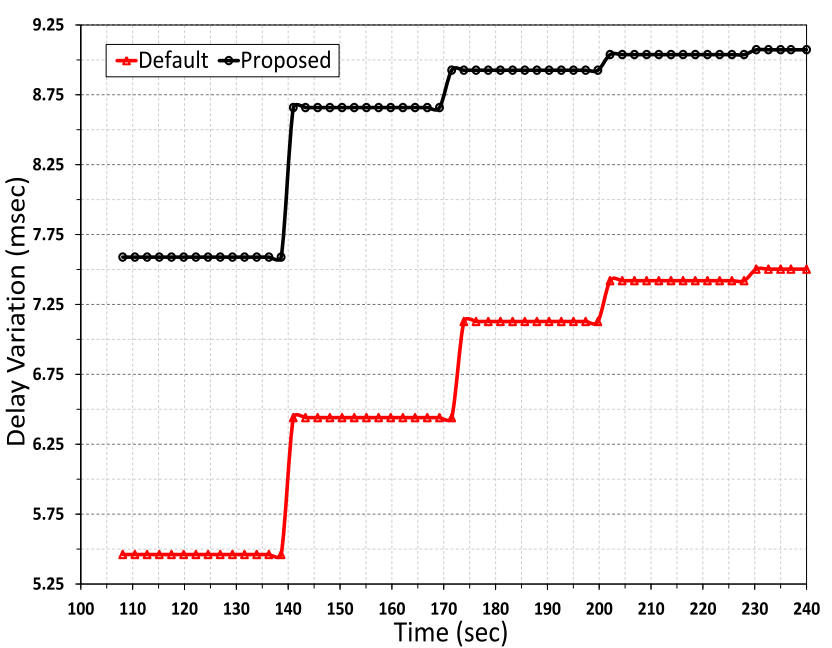

(b)

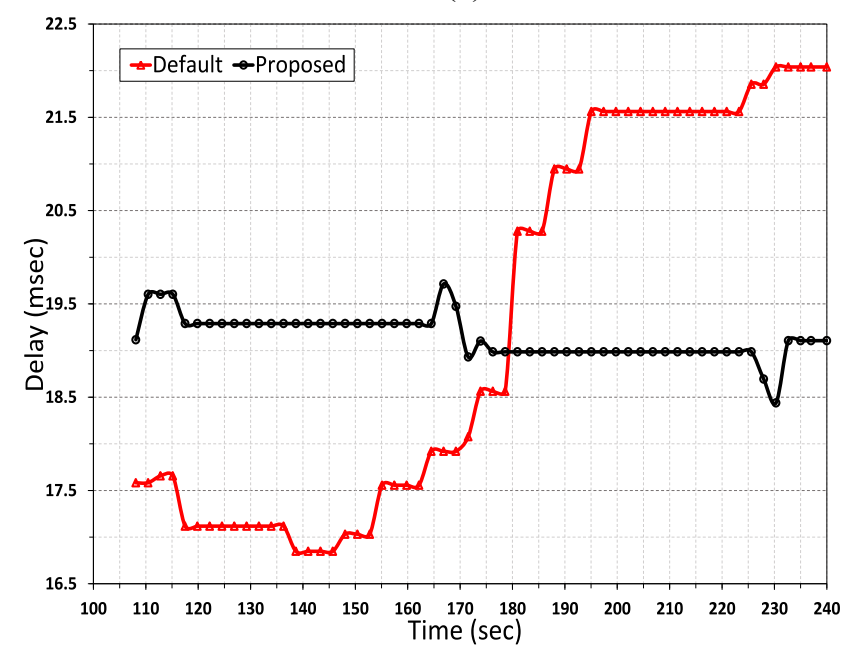

(d)

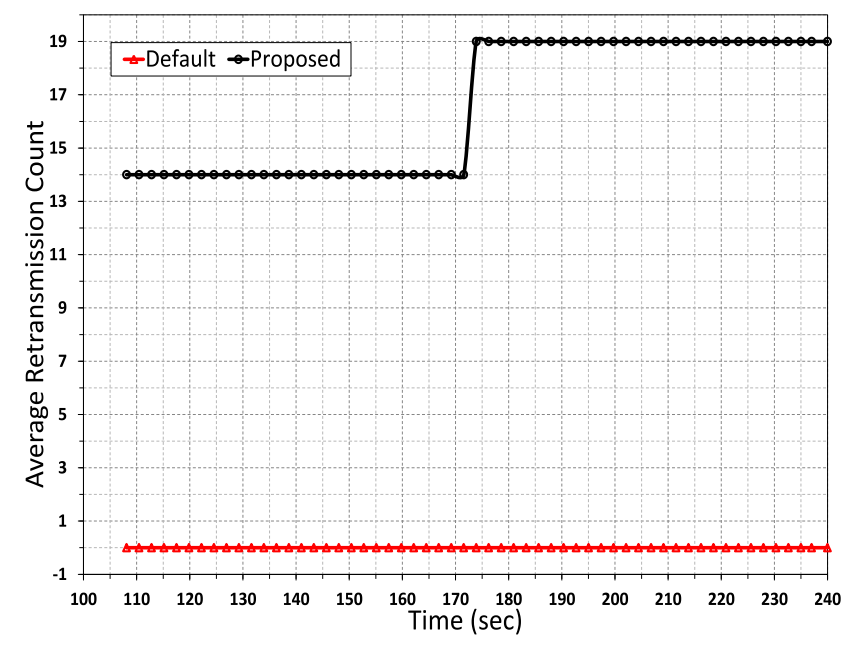

(f)

Fig. 10. Simulation results for the FTP and HTTP applications. (a) E2E packet delay for the FTP application. (b) PDV of the FTP application. (c) TCP retransmission count of the FTP application. (d) E2E packet delay for the HTTP application. (e) PDV of the HTTP application. (f) TCP retransmission count of the HTTP application.

The proposed network models average packet delay was between 19.6 and $18.6 \mathrm{~ms}$, whereas that for default network model was between 16.8 and $22 \mathrm{~ms}$. E2E delay variation is another important QoS factor for the HTTP service and is shown in Fig. 10(e). This figure shows the average E2E delay variation of received packets at the IP layer between one of the 
UEs and the HTTP service application server. The proposed network models delay variation was higher than for the default network model. The maximum average E2E delay variation of the former was $16.8 \mathrm{~ms}$, while that for the latter was $13.5 \mathrm{~ms}$.

Fig. 10(f) demonstrates the average TCP retransmission count with respect to simulation run time for the default and proposed network models. It shows that, in the default model, no retransmission happened during the HTTP application traffic flow. While in the proposed network model this did occur, which reflects that either there was a network congestion or a loss of packets received in the transport layer.

\section{DISCUSSION}

NQoS is a measure of the reliability and performance of the network nodes and links. It is a composite metric, being based on various factors that specify the characteristics of the network situation and consequently, deteriorations or improvements in the NQoS level can be brought about through some combined factors so as to fulfil AQoS requirements.

The proposed traffic management policy, with the assistance of WSN gateway, achieved high QoS, as presented by all the NQoS measurements of the MTC and HTC traffic flows in this paper at the IP and transport layers. The eNB performance achievement in terms of the UL and DL capacity enhancement was in PUSCH utilization, PUSCH throughput, and PDCCH utilization. In the proposed model the PUSCH utilization was reduced on average by $33.6 \%$ over the default model. The PUSCH throughput of the proposed model was achieved $73.2 \%$ increase over the default model, specifically when congestion was arisen. Another impact of the proposed flow management policy, it was on the PDCCH utilization achieve 5\% decrease at congestion condition over the default model. Furthermore, an increase of $179 \%$ in the radio access node connectivity, from the number of active connected MTDs point of view. The achieved NQoS enhancement was in terms of the radio access node physical layer performance parameters as well as of the MTC traffic flows exhibiting a decrease of $99.45 \%$ in the PLR, a decrease of $99.89 \%$ in the packet E2E delay, a decrease of $99.21 \%$ in the PDV.

Despite there being an increase of $12.42 \%$ with respect to the default baseline model occurred in terms of the VoLTE service as represented by its PLR, several offsetting achievements were delivered by the proposed traffic management policy, including a decrease of $9.5 \%$ in the PLR with respect to the 3GPP maximum PLR allowable limit for the VoLTE service, an increase of $4.5 \%$ from the baseline model for the packet E2E delay, a decrease of $18.79 \%$ from standard 3GPP maximum standard packet E2E delay, a reduction of $99.87 \%$ from the standard 3GPP maximum jitter value for the packet jitter and a decrease of $0.074 \%$ in terms of $\mathrm{QoE}$ from the default model values, with an increase of $8.73 \%$ from the standard values. The E2E VoLTE NQoS are the PLR, packet delay, and packet jitter, as a composite metrics. The degraded level of them can be improved by using a lower rate voice codec or adaptive multirate voice codec at the VoLTE application layer level [53]. It can be also improved by employing a scheduling algorithm at the physical layer level, specifically for VoLTE service bearer, such as TTI bundling and semi-persistent scheduling [54]. These scheduling techniques are exploiting processing delay difference between application layer voice frame generation process and physical layer transmission procedure [55].

Another NQoS improvement was for video conferencing, specifically at congestion condition, where a reduction of $85.75 \%$ regarding PLR, $85 \%$ of packet delay and $88.5 \%$ of PDV were achieved, when compared with the default model values.

The HTTP service NQoS achievement regarding packet E2E delay was a decrease of $13.31 \%$ and the PDV provided an increase of $37.26 \%$ to those of the baseline model. In spite of the obtained increment in the PDV, it represents a decrease of $52.26 \%$ from the standard maximum specified value.

FTP service NQoS delivered packet delay and delay variation increases of $9.12 \%$ and $20.9 \%$, respectively, when compared to the default model. Compared with the standard specified maximum allowable limits, the proposed policy achieved a rapid decrease of $95.36 \%$ in the packet delay and a reduction of $63.71 \%$ in delay variation.

Finally, the proposed model achieved the specified bearer settings for the HTDs and MTDs. Moreover, the QoE of the real time application users of the VoLTE service was maintained within the satisfaction level, as measured by the E-Model's MOS scale. While the QoE of the video conferencing service has been attained, in the perspective of obtainable NQoS metrics compared to the standard AQoS requirement of this service.

\section{CONCLUSION}

This paper has addressed the challenges of heterogeneity by integrating HTC and IoT traffic through their mutual interaction within network elements (nodes and links), in terms of the resultant and distinct E2E NQoS metrics. The motivation for this paper was based on the fact that the existing LTE-APro PCC rules and procedures do not differentiate between the user type of the terminal devices, which produces a lack of NQoS levels being offered and hence, potentially not meeting the AQoS demands made by the used service.

An access WSN gateway has been proposed to provide E2E connectivity for MTCs traffic flow within the license spectrum of LTE-APro media. An approach of protocol conversion and facilitation of the control of the EPS network resources QoS level assigned for MTC traffic flows, in the context of PCC functions and rules, has been provided. A policy for traffic flow management that defines MTC traffic flows over the EPS network has been put forward, in order to use a fraction of the available network resources in a seamless manner, accompanied by a new specific group of QCIs, assigned to define the QoS levels of the MTC bearers connectivity. The allocation of network resources for MTC traffic flows are tuned based on terminal type needs, i.e., on the level of importance of the traffic type sent by MTD. In addition, The QoE of real time application human users is assured within the perceived quality levels. 
The simulation results have shown that the proposed policy of traffic flow management within the LTE-APro network infrastructure outperforms the current one in terms of NQoS levels, not only for HTCs traffic flows but also, for those of MTCs. However, in terms of the VoLTE service, it some degradation was encountered with the offered NQoS, due to the use of high rate codec in the application layer and the absence of a specific scheduling procedure to the VoLTE bearer at the radio interface. Nevertheless, the VoLTE service QoE level was retained within the recommended standardization level.

The future work is matching the scope of this paper, and in the context of the heterogeneity of NGN, the key driver will be the use of network functions virtualization and software defined network to set the proposed policy in the network operating system layer as an engine to drive the control layer elements (controllers) in order to update the QoS mechanisms in the network elements, such as gateways in the $\mathrm{CN}$ as well as central offices in centralized RAN.

\section{REFERENCES}

[1] B.-W. Chen, W. Ji, F. Jiang, and S. Rho, "QoE-enabled big video streaming for large-scale heterogeneous clients and networks in smart cities,' IEEE Access, vol. 4, pp. 97-107, 2016.

[2] S. Yinbiao, P. Lanctot, and F. Jianbin, "Internet of Things: Wireless sensor networks," Geneva, Switzerland, Int. Electrotech. Commission, White Paper, 2014. [Online]. Available: http://www.iec.ch

[3] J. Eckenrode. (2015). The Derivative Effect: How Financial Services Can Make IoT Technology Pay Off. [Online]. Available: https:// dupress.deloitte.com/dup-us-en/focus/internet-of-things/iot-in-financialservices-industry.html?coll $=11711$

[4] F. Ghavimi and H.-H. Chen, "M2M communications in 3GPP LTE/LTEA networks: Architectures, service requirements, challenges, and applications," IEEE Commun. Surveys Tuts., vol. 17, no. 2, pp. 525-549, 2nd Quart., 2015.

[5] S. N. K. Marwat, T. Pötsch, Y. Zaki, T. Weerawardane, and C. Görg, "Addressing the challenges of E-healthcare in future mobile networks," in Proc. Meeting Eur. Netw. Universities Companies Inf. Commun. Eng., 2013, pp. 90-99.

[6] L. Costantino, N. Buonaccorsi, C. Cicconetti, and R. Mambrini, "Performance analysis of an LTE gateway for the IoT," in Proc. IEEE Int. Symp. World Wireless Mobile Multimedia Netw. (WoWMoM), 2012 pp. $1-6$.

[7] A. Lo, Y. W. Law, and M. Jacobsson, "A cellular-centric service architecture for machine-to-machine (M2M) communications," IEEE Wireless Commun., vol. 20, no. 5, pp. 143-151, Oct. 2013.

[8] D. Lee, J.-M. Chung, and R. C. Garcia, "Machine-to-machine communication standardization trends and end-to-end service enhancements through vertical handover technology," in Proc. IEEE 55th Int. Midwest Symp. Circuits Syst. (MWSCAS), 2012, pp. 840-844.

[9] S. Kang, W. Ji, S. Rho, V. A. Padigala, and Y. Chen, "Cooperative mobile video transmission for traffic surveillance in smart cities," Comput. Elect. Eng., vol. 54, pp. 16-25, Aug. 2016.

[10] S. N. K. Marwat, Y. Zaki, J. Chen, A. Timm-Giel, and C. Göerg, "A novel machine-to-machine traffic multiplexing in LTE-A system using wireless in-band relaying," in Proc. Int. Conf. Mobile Netw. Manag., 2013, pp. 149-158.

[11] J. Zhang, L. Shan, H. Hu, and Y. Yang, "Mobile cellular networks and wireless sensor networks: Toward convergence," IEEE Commun. Mag., vol. 50, no. 3, pp. 164-169, Mar. 2012.

[12] J.-P. Bardyn, T. Melly, O. Seller, and N. Sornin, "IoT: The era of LPWAN is starting now," in Proc. 42nd IEEE Eur. Solid State Circuits Conf. (ESSCIRC), 2016, pp. 25-30.

[13] D. Flore, 3GPP Standards for the Internet of Things, Qualcomm Technol. Inc., San Diego, CA, USA, 2016.

[14] E. Soltanmohammadi, K. Ghavami, and M. Naraghi-Pour, "A survey of traffic issues in machine-to-machine communications over LTE," IEEE Internet Things J., vol. 3, no. 6, pp. 865-884, Dec. 2016.

[15] R. P. Jover and I. Murynets, "Connection-less communication of IoT devices over LTE mobile networks," in Proc. 12th Annu. IEEE Int. Conf. Sens. Commun. Netw. (SECON), 2015, pp. 247-255.
[16] C. W. Johnson, LTE in Bullets. Northampton, U.K.: Chris Johnson, 2010.

[17] K. M. Koumadi, B. Park, and N. Myoung, "Introducing the latest 3GPP specifications and their potential for future AMI applications," KEPCO J. Elect. Power Energy, vol. 2, no. 2, pp. 245-251, 2016.

[18] I. Abdalla and S. Venkatesan, "A QoE preserving M2M-aware hybrid scheduler for LTE uplink," in Proc. IEEE Int. Conf. Sel. Topics Mobile Wireless Netw. (MoWNeT), 2013, pp. 127-132.

[19] L. Galluccio, S. Milardo, G. Morabito, and S. Palazzo, "SDN-WISE: Design, prototyping and experimentation of a stateful SDN solution for wireless sensor networks," in Proc. IEEE Conf. Comput. Commun. (INFOCOM), 2015, pp. 513-521.

[20] Digital Cellular Telecommunications System (Phase 2+) (GSM), Universal Mobile Telecommunications System (UMTS); LTE; Policy and Charging Control Architecture, ETSI Standard TS 123203 V13.6.0 (2016-03), 2016.

[21] M. Chiang and T. Zhang, "Fog and IoT: An overview of research opportunities," IEEE Internet Things J., vol. 3, no. 6, pp. 854-864, Dec. 2016.

[22] N. Seitz, "ITU-T QoS standards for IP-based networks," IEEE Commun. Mag., vol. 41, no. 6, pp. 82-89, Jun. 2003.

[23] "Network performance objectives for IP-based services," Int Telecommun. Union, Geneva, Switzerland, ITU Recommendation Y.1541, Feb. 2006.

[24] O. Modeler. (2016). Riverbed Technology Inc. [Online]. Available: http://www.riverbed.com

[25] H. Ekstrom, "QoS control in the 3GPP evolved packet system," IEEE Commun. Mag., vol. 47, no. 2, pp. 76-83, Feb. 2009.

[26] S. Albasheir and M. Kadoch, "Enhanced control for adaptive resource reservation of guaranteed services in LTE networks," IEEE Internet Things J., vol. 3, no. 2, pp. 179-189, Apr. 2016.

[27] N. A. Ali, A.-E. M. Taha, and H. S. Hassanein, "Quality of service in 3GPP R12 LTE-advanced," IEEE Commun. Mag., vol. 51, no. 8, pp. 103-109, Aug. 2013

[28] L. Korowajczuk, How to Dimension User Traffic in $4 G$ Networks (CelPlan Webinar Series). Webinar, Jun. 2014. [Online]. Available: http://www.celplan.com/webinars/webinar-20140507.asp

[29] X. An, F. Pianese, I. Widjaja, and U. G. Acer, "DMME: A distributed LTE mobility management entity," Bell Labs Tech. J., vol. 17, no. 2, pp. 97-120, 2012.

[30] G. Crosby and F. Vafa, "A novel dual mode gateway for wireless sensor network and LTE-A network convergence," Int. J. Eng. Res. Innovat., vol. 5, no. 2, pp. 19-27, 2013

[31] G. Horváth, "End-to-end QoS management across LTE networks," in Proc. IEEE 21st Int. Conf. Softw. Telecommun. Comput. Netw. (SoftCOM), 2013, pp. 1-6.

[32] "LTE in a nutshell: Protocol architecture," Markham, ON, Canada, Telesystem Innovations, White Paper, 2010.

[33] A. M. Ghaleb, E. Yaacoub, and D. Chieng, "Physically separated uplink and downlink transmissions in LTE HetNets based on CoMP concepts," in Proc. Int. Conf. Front. Commun. Netw. Appl. (ICFCNA Malaysia), 2014, pp. 1-6.

[34] H. Holma and A. Toskala, WCDMA for UMTS: HSPA Evolution and LTE. Chichester, U.K.: Wiley, 2010.

[35] Cisco. (2016). Voice Over IP-Per Call Bandwidth Consumption. [Online]. Available: http://www.cisco.com/c/en/us/ support/docs/voice/voice-quality/7934-bwidth-consume.html

[36] S. J. M. Baygi and M. Mokhtari, "Evaluation performance of protocols LEACH, 802.15.4 and CBRP, using analysis of QoS in WSNs," Wireless Sensor Netw., vol. 6, no. 10, pp. 221-236, 2014.

[37] S. O. Robert. (2014). Lectures: CSCI 4151 Systems Simulation Class. [Online]. Available: http://www.robertowor.com/csci4151/lecture5.htm

[38] N. Ince and A. Bragg, Recent Advances in Modeling and Simulation Tools for Communication Networks and Services. New York, NY, USA: Springer, 2007. [Online]. Available: https://books.google.co.uk/books?id=pJSqZZmc9_oC

[39] Riverbed_Technology. (2017). Verifying Statistical Validity of Discrete Event Simulations. [Online]. Available: http://enterprise14.opnet.com/ 4dcgi/CL_SessionDetail?ViewCL_SessionID=3172,White_Paper_from_ OPNET Verifying_Statistical_Validity_DES.pdf

[40] (2007). OpNet Modeling Concepts. [Online]. Available: http://suraj. lums.edu.pk/te/simandmod/Opnet/07\%20Simulation\%20Design.pdf

[41] Rohde\&Schwarz. (2017). 1MA272: Testing LTE-A Releases 11 and 12. [Online]. Available: https://www.rohde-schwarz.com/us/ applications/testing-lte-a-releases-11-and-12-application-note_56280307009.html 
[42] Evolved Universal Terrestrial Radio Access (E-UTRA); Physical Layer Procedures, 3GPP Standard TS 36.213 V14.2.0 (2017-03), 2017. [Online]. Available: https://portal.3gpp.org/desktopmodules/ Specifications/SpecificationDetails.aspx? specificationId $=2427$

[43] A. Sethi and V. Hnatyshin, The Practical OPNET User Guide for Computer Network Simulation. Boca Raton, FL, USA: Taylor \& Francis, 2012. [Online]. Available: https://books.google.co.uk/ books?id=3E3wqbSoHQQC

[44] "One voice; voice over IMS profile, V1.0.0," AT\&T, Dallas, TX, USA, Orange, Paris, France, Telefonica, Madrid, Spain, TeliaSonera, Stockholm, Sweden, Verizon, Basking Ridge, NJ, USA, Vodafone, London, U.K., Alcatel-Lucent, Boulogne-Billancourt, France, Ericsson, Stockholm, Sweden, Nokia Siemens Networks, Nokia, Espoo, Finland, Samsung, Seoul, South Korea, and Sony Ericsson, Tokyo, Japan, Nov. 2009. [Online]. Available: http://cdnet.stpi.narl.org.tw/techroom/ market/_pdf/2009/eetelecomm_09_009_OneVoiceProfile.pdf

[45] Technical Specification Group Services and System Aspects; Policy and Charging Control Architecture (Release 10), Volume 10.6.0, 3GPP Standard TS 23.203, Mar. 2012.

[46] M. R. Tabany and C. G. Guy, "An end-to-end QoS performance evaluation of VoLTE in 4G E-UTRAN-based wireless networks," in Proc. 10th Int. Conf. Wireless Mobile Commun., 2014, pp. 90-97.

[47] Cisco. (2005). Cisco Networking Academy Program: IP Telephony v1.0. [Online]. Available: http://www.hh.se/download/ 18.70cf2e49129168da015800094781/1341267715838/7_6_Jitter.pdf

[48] S. A. Ahson and M. Ilyas, VoIP Handbook: Applications, Technologies, Reliability, and Security, Boca Raton, FL, USA: CRC Press, 2008.

[49] The E-model: A computational model for use in transmission planning, Int. Telecommun. Union, Geneva, Switzerland, ITU Recommendation G.107, Jun. 2015. [Online]. Available: https://www.itu.int/rec/T-RECG.107-201506-I/en

[50] A. Clark and B. Claise. (2011). Guidelines for Considering New Performance Metric Development. [Online]. Available: https://tools.ietf.org/html/rfc6390.html

[51] A. D. Clark, "Modeling the effects of burst packet loss and recency on subjective voice quality," in Proc. Columbia Univ. IP Telephony Workshop, 2001.

[52] B. Belmudez, Audiovisual Quality Assessment and Prediction for Videotelephony (T-Labs Series in Telecommunication Services). Cham, Switzerland: Springer, 2014. [Online]. Available: https://books.google.co.uk/books?id=ULTzBQAAQBAJ

[53] IMS Profile for Voice and SMS, Version 9.0, document. IR. 92, GSM Assoc., London, U.K., 2015.

[54] VoLTE White Paper, China Mobile, Hong Kong, 2013.

[55] "Deployment and the radio access network: The LTE user equipment perspective," Sunnyvale, CA, USA, Spirent, White Paper, 2012.

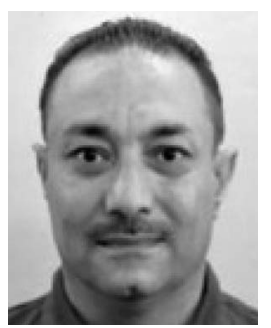

Basim K. J. Al-Shammari (GS'15) received the B.Sc. Eng. degree in electrical and electronic engineering/communications and electronic warfare from the Military College of Engineering, Baghdad, Iraq, in 1991, and the M.Sc. Eng. degree in electrical and electronic engineering/communications from the Al-Rasheed College of Engineering \& Science, Baghdad, in 2005. He is currently pursuing the Ph.D. degree in communications engineering at Brunel University London, London, U.K.

His current research interests include antenna, propagation, communication system design, QoS, QoE, mobile network deployment design, CRAN, NFV, MTC, wireless SDN, and smart IoT services and applications.

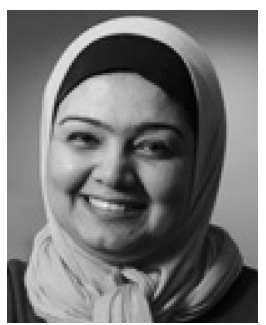

Nadia Al-Aboody (GS'14-M'16) received the B.Sc. degree in software engineering from Al-Mansour University College, Baghdad, Iraq, in 2003 and the master's degree in computer networking from the Iraqi Commission for Computer and Informatics, Baghdad, in 2007. She is currently pursuing the $\mathrm{Ph} . \mathrm{D}$. degree in communications engineering at Brunel University London, London, U.K.

Her current research interests include Internet of Things, machine to machine, and wireless sensor networks.

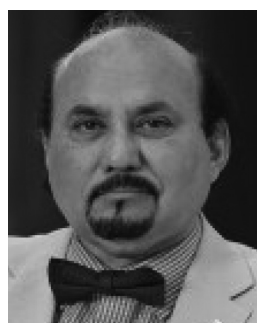

Hamed S. Al-Raweshidy (M'92-SM'97) received the B.Eng. and M.Sc. degrees in communication engineering from the University of Technology, Baghdad, Iraq, in 1977 and 1980, respectively, the Postgraduate Diploma degree from Glasgow University, Glasgow, U.K., in 1987, and the Ph.D. degree from Strathclyde University, Glasgow, in 1991.

$\mathrm{He}$ is a Professor of communication engineering with the University of Technology. He was with the Space and Astronomy Research Centre, Baghdad, PerkinElmer, Waltham, MA, USA, Carl Zeiss, Oberkochen, Germany, British Telecom, Edinburgh, U.K., Oxford University, Oxford, U.K. Manchester Metropolitan University, Manchester, U.K., and Kent University, Canterbury, U.K. He is currently the Director of the Wireless Networks and Communications Centre, Brunel University, London, U.K. He has authored or co-authored over 250 papers in international journals and referred conferences. His current research interests include radio over fiber, personal networks, spectral efficiency, green radio, cognitive communications, and mesh networks.

Prof. Al-Raweshidy has been a Guest Editor for the International Journal of Wireless Personal Communications. He is an Editorial Board member of several journals such as the Journal of Communications and Mobile Computing and the Journal of Wireless Personal Communications. He is the Chairman of Expert Working Group 4, Wired and Wireless Integration for Future Network, in eMobility, which is the top technology platform in Europe and it advises the European Commission for research directions in communications for the next 10-15 years. He is a member of the Senate for Brunel University and the School of Engineering Research Committee. He is a college member for EPSRC/U.K. and a Reviewer for the EU Commission, Hong Kong, and Cyprus. 